\title{
Transcriptional Regulation of the Glutamate/GABA/Glutamine Cycle in Adult Glia Controls Motor Activity and Seizures in Drosophila
}

\author{
-David Mazaud, ${ }^{1}$ Benjamin Kottler, ${ }^{1}{ }^{-}$Catarina Gonçalves-Pimentel, ${ }^{1}$ Sandra Proelss, ${ }^{1}$ Nadine Tüchler, ${ }^{1}$ \\ CCeline Deneubourg, ${ }^{1}$ Yoshihiro Yuasa, ${ }^{2,3,4,5}$ Celine Diebold, ${ }^{2,3,4,5}$ Heinz Jungbluth, ${ }^{1}{ }^{\circ E}$ Eric C. Lai, ${ }^{6}$ 'Frank Hirth, ${ }^{1}$ \\ Angela Giangrande, ${ }^{2,3,4,5}$ and ${ }^{\circ}$ Manolis Fanto ${ }^{1}$ \\ ${ }^{1}$ Department of Basic and Clinical Neuroscience, King's College London, SE5 9NU London, United Kingdom, ${ }^{2}$ Institut de Génétique et de Biologie \\ Moléculaire et Cellulaire, 67404 Illkirch, France, ${ }^{3}$ Centre National de la Recherche Scientifique, UMR7104, Illkirch, France, ${ }^{4}$ Institut National de la Santé et \\ de la Recherche Médicale, U1258, Illkirch, France, ${ }^{5}$ Université de Strasbourg, 67404 Illkirch, France, and ${ }^{2}$ Department of Developmental Biology, Sloan- \\ Kettering Institute, New York, New York 10065
}

The fruitfly Drosophila melanogaster has been extensively used as a genetic model for the maintenance of nervous system's functions. Glial cells are of utmost importance in regulating the neuronal functions in the adult organism and in the progression of neurological pathologies. Through a microRNA-based screen in adult Drosophila glia, we uncovered the essential role of a major glia developmental determinant, repo, in the adult fly. Here, we report that Repo expression is continuously required in adult glia to transcriptionally regulate the highly conserved function of neurotransmitter recycling in both males and females. Transient loss of Repo dramatically shortens fly lifespan, triggers motor deficits, and increases the sensibility to seizures, partly due to the impairment of the glutamate/GABA/glutamine cycle. Our findings highlight the pivotal role of transcriptional regulation of genes involved in the glutamate/GABA/glutamine cycle in glia to control neurotransmitter levels in neurons and their behavioral output. The mechanism identified here in Drosophila exemplifies how adult functions can be modulated at the transcriptional level and suggest an active synchronized regulation of genes involved in the same pathway. The process of neurotransmitter recycling is of essential importance in human epileptic and psychiatric disorders and our findings may thus have important consequences for the understanding of the role that transcriptional regulation of neurotransmitter recycling in astrocytes has in human disease.

Key words: Drosophila; glia; glutamate/GABA/glutamine; repo

\section{Significance Statement}

Glial cells are an essential support to neurons in adult life and have been involved in a number of neurological disorders. What controls the maintenance and modulation of glial functions in adult life is not fully characterized. Through a miR overexpression screen in adult glia in Drosophila, we identify an essential role in adult glia of repo, which directs glial differentiation during embryonic development. Repo levels modulate, via transcriptional regulation, the ability of glial cells to support neurons in the glutamate/GABA/glutamine cycle. This leads to significant abnormalities in motor behavior as assessed through a novel automated paradigm. Our work points to the importance of transcriptional regulation in adult glia for neurotransmitter recycling, a key process in several human neurological disorders.

\section{Introduction}

Glial cells constitute a significant part of the nervous system and are devoted to a variety of functions of essential importance for the correct functioning of neurons, including a fundamental role in uptake and recycling of neurotransmitters (Rae et al., 2003). Therefore, glial cells remain of paramount importance through- 
out life to allow proper homeostasis of the nervous system, as evidenced by the association of glial functions' failures with neurological pathologies (Lynch et al., 2010; Furrer et al., 2011).

Glia in Drosophila provides many of the same functions as in mammals, including regulation of neurotransmitter uptake and recycling (Rival et al., 2004; Chaturvedi et al., 2014). We have performed a genetic screen to identify genetic elements required for glial function by specifically deregulating gene expression only in the adult Drosophila glia to avoid any developmental effect, and have used miRNAs to uncover key regulatory modules. The promiscuous nature of miRNAs allows to target and finetune the expression of several target genes (Lim et al., 2005; Bartel, 2009; Kozomara and Griffiths-Jones, 2011). In specific cell types and at specific time points, one or more of the miRNAs targets acquire special importance and can be singled out as the cause for the phenotype elicited by specific miRNAs (Silver et al., 2007; Bejarano et al., 2012).

Behavioral activity is the outcome of neural interactions mediated by different circuits. We hypothesized that glia manipulations will lead to a variety of behavioral consequences and structured our approach in three steps that allow detection of the progressive contribution of the genetic manipulations to different behavioral manifestations. First, we used fly lifespan as an unrefined readout. We then characterized endogenous and exogenous behavioral activity by simple motor readout (negative geotaxis) and, further, by adapting video-tracking methodology with control of mechanical stimulus. Using this novel approach allows detection of the progressive contribution of the genetic manipulations to different behavioral manifestations.

Here, we report that overexpression of miR-1 in adult glial cells shortens the fly lifespan, partially through the downregulation of Repo, a key transcription factor for the development of almost all Drosophila glia (Xiong et al., 1994; Halter et al., 1995). This led to the identification of a major regulatory module in Drosophila adult glial cell function maintenance, which points to the importance of transcriptional regulation of neurotransmitter recycling in glia with possible consequences for human neurological and psychiatric disorders.

\section{Materials and Methods}

Drosophila stocks and husbandry. Flies were maintained at either $18^{\circ} \mathrm{C}$ or room temperature on standard cornmeal agar medium $(0.8 \% \mathrm{w} / \mathrm{v}$ agar, $2 \% \mathrm{w} / \mathrm{v}$ cornmeal, $8 \% \mathrm{w} / \mathrm{v}$ glucose, $5 \% \mathrm{w} / \mathrm{v}$ Brewer's yeast, $1.5 \%$ $\mathrm{v} / \mathrm{v}$ ethanol, $0.22 \% \mathrm{v} / \mathrm{v}$ methyl-4-hydroxybenzoate, $0.38 \% \mathrm{v} / \mathrm{v}$ propionic acid). All of the following lines were obtained from the Bloomington collection: $w^{1118}$ (RRID:BDSC_3605), repoGal4 (RRID: BDSC_7415), dEAAT1Gal4 (RRID:BDSC_8849), NP2222Gal4 (RRID:DGGR_112830), MZ0709Gal4, moodyGal4, GMRGal4 (RRID: BDSC_9146), Actin5cGal4 (RRID:BDSC_4414), elavGal4 (RRID: BDSC_8765), ubiGal80 ${ }^{\text {ts }}$, tubGal80 ${ }^{\text {ts }}$ (RRID:BDSC_7019), UASdEAAT1 (RRID:BDSC_8202), UAS-iGluSnFRA184S (RRID: BDSC_59610), and UAS-repo ${ }^{\text {IR }} 2$ (TRiP.JF 02974 RRID:

Association pour la Recherche sur le Cancer (A.G.), Agence Nationale de la Recherche (A.G.), and by Grant ANR-10LABX-0030-INRT, a French State fund managed by the Agence Nationale de la Recherche under the frame program Investissements d'Avenir ANR-10-IDEX-0002-02. E.C.L. was supported by the National Institutes of Health (Grant R01-NS083833 and MSK Core Grant P30-CA008748). We thank M. Freeman, R. Ordway, A. Delogu, M. Meyer, the VDRC, the DSHB, and the BDSC for fly stocks and reagents; Alix Gaultier De La Ferriere for technical assistance; and R. Sousa-Nunes and 0 . Baron for comments on this manuscript.

B.K. is cofounder of BFK Lab. The remaining authors declare no competing financial interests.

Correspondence should be addressed to Manolis Fanto at manolis.fanto@kcl.ac.uk.

https://doi.org/10.1523/JNEUROSCI.1833-18.2019

Copyright $\odot 2019$ Mazaud et al.

This is an open-access article distributed under the terms of the Creative Commons Attribution License Creative Commons Attribution 4.0 International, which permits unrestricted use, distribution and reproduction in any medium provided that the original work is properly attributed.
BDSC_28339). alrmGal4 (RRID:BDSC_67031) and UAS-Gat were kindly provided by M. Freeman. UAS-repo ${ }^{\mathrm{IR}} 1$ (GD 10424, RRID: FlyBase_FBst0450092) was obtained from the VDRC collection, UASrepo-myc was described previously (Matsuno et al., 2015), UAS-repo was also described previously (Yuasa et al., 2003), and repo- $n G F P$ was generated by C. Diebold. UAS-miR-1 was generated by E. Lai for the miRNA library (Bejarano et al., 2012). UAS-EGFP-Gs2 was kindly provided by R.W. Ordway.

Lifespan. Lifespan analysis was performed as described previously (Nisoli et al., 2010). Briefly, all crosses were maintained at $18^{\circ} \mathrm{C}$ during the developmental stages of the progeny. Newly eclosed adult flies were collected within $5 \mathrm{~d}$ at $18^{\circ} \mathrm{C}$. Females and males were pooled together and equally distributed within three vials. Sixty flies were assessed unless specified otherwise. All lifespan analysis was done in a controlled environment of $29^{\circ} \mathrm{C}$ and $60 \%$ humidity or $25^{\circ} \mathrm{C}$ when specifically stated. Using $\mathrm{CO}_{2}$ to anesthetize the live flies, those dead and alive were counted and live ones transferred into fresh vials three times per week. For the lifespans done with only $3 \mathrm{~d}$ at $29^{\circ} \mathrm{C}$ before being transferred to $18^{\circ} \mathrm{C}$, flies were still counted 3 times per week but were transferred into a fresh vial only once a week.

Climbing assay. Flies were collected from the same cross used for the lifespan experiment to assess their negative geotaxis reaction. Ten female flies from each genotype were transferred into $70 \mathrm{~mm}$ tubes. The tubes containing compared genotype were assessed at the same time using a custom-made array. Four sessions were recorded to evaluate the vertical position of each fly after $1 \mathrm{~min}$. The scores from the 10 females were averaged for each repeat and the average of the four repeats calculated.

Automatic motor behavioral assay. Single fly tracking was performed as described previously (Faville et al., 2015). In each experiment, $\sim 20 \mathrm{fe}-$ male flies per genotype were anesthetized on ice and individually placed into glass tubes. All of the genotypes were positioned on the same platform, with two shaftless motors placed under each subplatform containing each one genotype. The flies were allowed to recover for $30 \mathrm{~min}$ at $25^{\circ} \mathrm{C}$ before the start of the procedure. The protocol used for the stimuli response is presented with a schematic in Figure $7 A$. In summary, 6 stimuli events were equally split during a period of $2 \mathrm{~h}$ and $15 \mathrm{~min}$, the first one starting after $30 \mathrm{~min}$ of recording and the last one $30 \mathrm{~min}$ before the end of the protocol. Each stimuli event was composed of five vibrations of $200 \mathrm{~ms}$ spaced by $500 \mathrm{~ms}$. The $x / y$ position of each single fly was tracked and analyzed using Drosophila ARousal Tracking (DART) software (Faville et al., 2015) to evaluate the relative speed and activity before, during and after the stimuli event. The speed analysis is used for the "stimuli response trace" and the general activity was used to deduce "active speed," "mean bout length," and "interbout interval" (for details, see Kottler et al., 2017) using a custom-made modification of the DART software (Faville et al., 2015). The DART-derived graphs were edited with Adobe Illustrator CC2017 (RRID:SCR_010279).

Heat-induced seizure assay. The heat-induced seizure assay was adapted from one described previously (Sun et al., 2012). Ten flies per genotype were isolated into plastic vials with food $3 \mathrm{~h}$ before the assay. They were then allowed to accommodate into new plastic vials without food for 10-20 min before immersion in a $40^{\circ} \mathrm{C}$ water bath for $2 \mathrm{~min}$. Each tube was video recorded during and after immersion and seizures were defined as a period of brief leg twitches and failure to maintain standing posture. The mean of the time to recover from seizure was calculated for each genotype. The experiment was repeated five times independently and averaged.

Adult brain staining. Adult brain staining was performed as described previously (Baron et al., 2017). Briefly, flies were anesthetized on ice. Brains were dissected and put straight into $4 \%$ paraformaldehyde (PFA) for $45 \mathrm{~min}$ of fixation. They were then washed in phosphate buffer saline solution with $0.1 \%$ Tween (PBS-T) for 30 min before blocking in $5 \%$ bovine serum albumin (BSA) in PBS-T for $1 \mathrm{~h}$ at room temperature (RT) in a 96-well plate. Primary and secondary antibodies were diluted in blocking solution and incubated overnight at $4^{\circ} \mathrm{C}$. After three washes of $15 \mathrm{~min}$, brains were mounted in Vectashield on a slide surrounded by two coverslips on each side before being covered by another coverslip on top (to prevent the brains from being crushed). The antibodies were used as follows: anti-GFP (1/100, mouse, Roche, RRID:AB_390913), anti- 
Table 1. Oligos and UPLs used for qPCR

\begin{tabular}{|c|c|c|c|c|}
\hline Gene & CGno. & UPL probe no. & Left oligo & Right oligo \\
\hline 1000 & CG5248 & 116 & CTGGTTTATCAACGCCTATGAA & GAGTGCGGAAGGAAGACTGT \\
\hline Gliotactin & CG3903 & 69 & CGAATCGTCCAATTACAGAGC & GAAAAATTCCAGGAGAAACTGG \\
\hline GS2 & CG1743 & 127 & GCACCCTCGACTTCATTCC & GCACGAGCTTCCATCGTAGT \\
\hline Pointed & CG17077 & 63 & CTTTCTGTCCAGCCTAGTTGAGT & TGCACAGATCCTTGCATCC \\
\hline dEAAT1 & CG3747 & 30 & GAATAAATTTGCTTGACATCCTTTT & AAAGCACGATTGGCAGTCA \\
\hline$w g$ & CG4889 & 81 & GGCAAAATCGTTGATCGAG & GCAGGACTCTATCGTTCCTTCA \\
\hline Gat & CG1732 & 31 & TTCTTTATGTCGATGAGAGCAGA & СCTTTCATATTGACTGACACAGTTG \\
\hline Gapdh & CG12055 & 18 & AAAAAGCTCCGGGAAAAGG & AATTCCGATCTTCGACATGG \\
\hline elF4A & CG9075 & 104 & CGTGAAGCAGGAGAACTGG & СATCTCCTGGGTCAGTTGGT \\
\hline
\end{tabular}

Shown are sequences of oligos used for qPCR and probe numbers from the Universal Probe Library given for each gene tested.

A

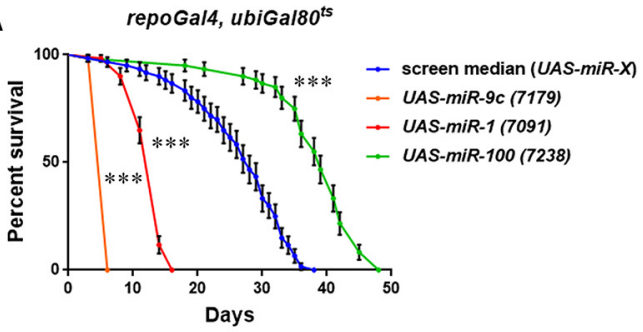

B Effect of glial miRNAs overexpression on lifespan

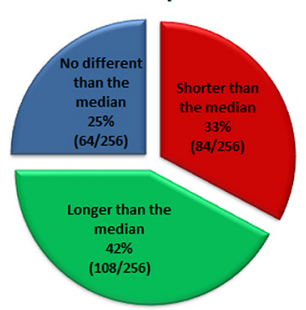

D

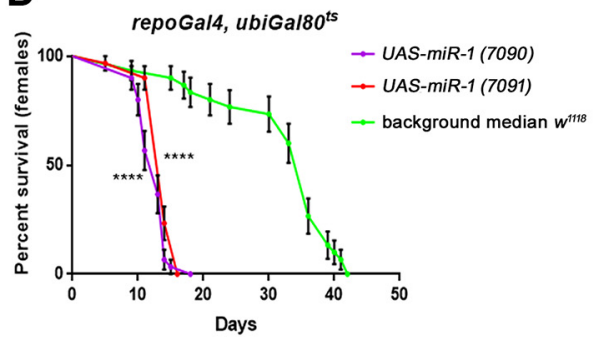

$\mathbf{F}$

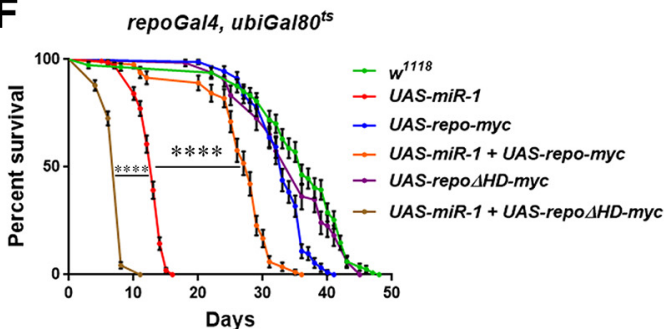

Figure 1. Effect of miRNA expression in adult Drosophila glia. All aging in this figure was performed at $29^{\circ} \mathrm{C}$. $A$, Examples of short-lived (UAS-miR-9 and UAS-miR-1) and long-lived (UAS-miR-100) miRNA lines overexpressed in adult glial cells compared with the screen median lifespan. Each lifespan was done with 60 flies (30 females and 30 males; see Materials and Methods). Log-rank (Mantel-Cox) test $p<0.000000000001$ for all. For a full screen report, see Figure 1-1 (available at https://doi.org/10.1523/JNEUROSCI.1833-18.2019.f1-1). B, Summary of the miRNA screen. Each line is compared with the median lifespan of the library (256 lines from 133 miRNAs). C, Comparison of the median from $6 \mathrm{w}^{1118}$; ubi-Gal80 $/$ ts + ; repoGal4/+ lifespan with the median lifespan of the screen $w^{1118}$; (ubi-Gal80 ${ }^{\text {ts }}$; repoGal4)/UAS-miR-X. Log-rank (Mantel-Cox) test $p<0.0001$ (D) Lifespan of female flies expressing UAS-miR-1, from an autosomal insertion (7091) and from an insertion on the X chromosome (7090) compared with female median $w^{1118}$ controls. Both display a dramatic reduction in lifespan. Log-rank (Mantel-Cox) test $p<0.0001$ for both. $\boldsymbol{E}$, Western blot analysis of Repo protein level upon miR-1 overexpression in adult glia. There is a strong reduction of Repo protein compared with control, quantified on the right panel (Mann-Whitney, $n=3, p=$ 0.049). The genotypes were $w^{1118}$; ubi-Gal80 ${ }^{\text {ts }} /+$; repoGal4/+ and $w^{1118}$; ubi-Gal80 ${ }^{\text {ts }} /+$; repoGal4/UAS-miR-1 (7091). $\boldsymbol{F}$, Rescue of UAS-miR-1 short lifespan by coexpression of UAS-repo-myc (log-rank Mantel-Cox test $p<0.000000000001)$, which by itself displays instead a mildly decreased lifespan compared with controls. Conversely, the coexpression of a mutated dominantnegative repo transgene, UAS-repo $\triangle H D$-myc, enhanced the shortening of lifespan due to miR-1 overexpression (log-rank Mantel-Cox test $p<0.0001$ ), ruling out any weakening of the UAS-miR-1 effect due to a second UAS-based transgene, whereas it had no effect of its own when expressed alone. The driver used was repoGal4, ubi-Gal80 ${ }^{\text {ts }}$. Between 80 and 180 female flies were assessed. ${ }^{*} p<0.05,{ }^{* * *} p<0.001,{ }^{* * * *} p<0.0001$.

GFP (1/500, chicken, kindly provided by M. Meyer), anti-Repo (1/100, mouse DSHB 8D12, RRID:AB_528448), anti-Elav (1/500, rat, DSHB 758A10, RRID:AB_528218 or 1/2000 mouse, DSHB, 9F8A9, RRID: AB_528217), and anti-GABA (1/1000, Sigma-Aldrich A2052, RRID: AB_477652 kindly provided by A. Delogu).

iGluSnFRA184S imaging. Flies expressing the glutamate sensor iGluSnFRA184S were anesthetized on ice. The flies developed at $18^{\circ} \mathrm{C}$ and were transferred to $29^{\circ} \mathrm{C}$ for $7 \mathrm{~d}$ once they reached the adult stage.
Brains were dissected into Schneider's medium and transferred directly into a dish filled with Schneider's medium to be imaged. Confocal pictures of each brain were taken within $20 \mathrm{~min}$ after dissection. $Z$-stacks of $1 \mu \mathrm{m}$ sections were taken for each genotype. The microscope settings were established using control flies to have a GFP signal below saturation and kept unchanged throughout all acquisitions. These photographs were taken with a Nikon Spinning Disc confocal microscope and analyzed with ImageJ Fiji software (RRID:SCR_002285). A square of $166 \times$ 
A

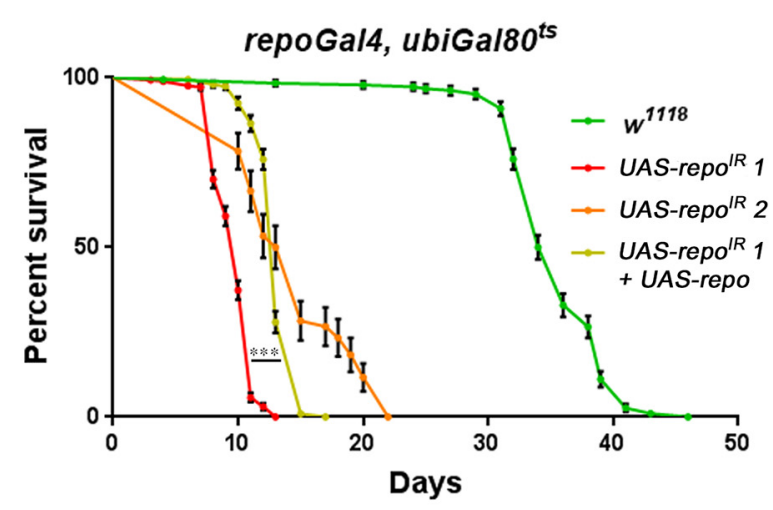

B

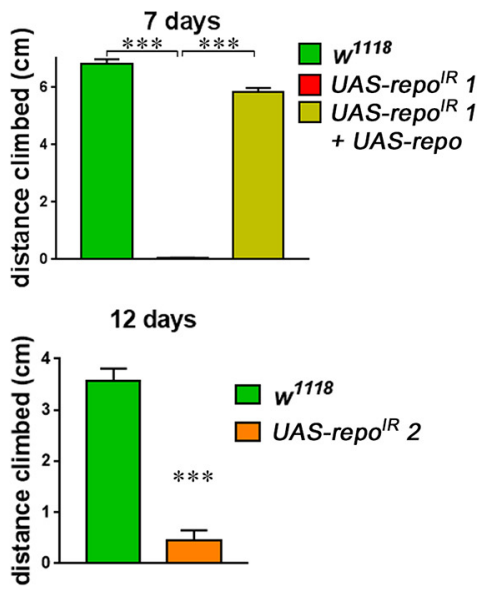

D

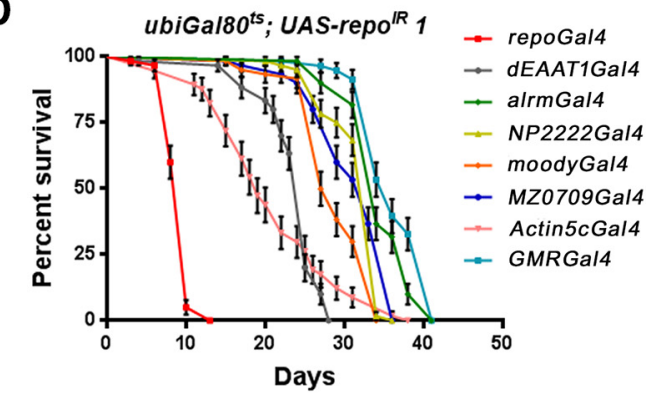

Figure 2. Repo protein levels influence Drosophila lifespan and behavior. All aging in this figure was performed at $29^{\circ} \mathrm{C}$. $\boldsymbol{A}$, Lifespan of two different RNAi lines against repo and the rescue of the RNAi 1 by coexpression of a UAS-repo transgene (log-rank Mantel-Cox test $p<0.0001$ ). $\boldsymbol{B}$, Climbing assay of the two RNAi lines against repo and the rescue of the RNAi 1 by UAS-repo as assessed just before death (respectively 7 for RNAi 1 , one-way ANOVA, Tukey's multiple-comparisons test $p=0.0001$ and $p<0.0001$, respectively, and $12 \mathrm{~d}$ for RNAi 2 at $29^{\circ} \mathrm{C}$, Mann-Whitney, $n=3, p=$ 0.049). C, Western blot analysis of Repo levels after $7 \mathrm{~d}$ at $29^{\circ} \mathrm{C}$. D, Sixty flies ( 30 females and 30 males) were used for each genotype. Only repo knock-down by the ubiquitous $A$ ctin 5 cGal 4 or the mosaic dEAAT1-Gal4 achieved a significant detrimental effect on fly lifespan, supporting the composite nature of the effect on lifespan. The specificity of each driver is as follows: repoGal4 (pan-glial), alrmGal4 (astrocyte-like glia), NP2222Gal4 (cortex glia), moodyGal4 (subperineurial glia), MZ0709Gal4 (ensheathing glia), Actin5cGal4 (ubiquitously expressed), dEAAT1Gal4 (astrocyte-like glia, cortex glia and some subperineurial glia), and GMRGal4 (eye, used as negative control, not expressed in glial cells). ${ }^{* * *} p<0.001$.

166 pixels was drawn around the calyx region and the intensity was measured. The three highest values of each $z$-stack were averaged. At least three different calyx regions were measured per genotype.

TUNEL assay. Fly brains were dissected in cold PBS and fixed for 45 min in $4 \%$ PFA at RT. After three washes of $10 \mathrm{~min}$ in PBS, the brains were incubated $10 \mathrm{~min}$ in $50 \mu \mathrm{l}$ of proteinase $\mathrm{K}(20 \mu \mathrm{g} / \mathrm{ml}$ in PBS $)$ and then washed twice for $10 \mathrm{~min}$ in PBS (to stop the permeabilization). For the positive control, a 10 min incubation in DNase I buffer was followed by a $10 \mathrm{~min}$ incubation in DNase I ( $7 \mathrm{U} / \mathrm{ml}$ in DNase I buffer) and then washed three times in double-distilled water $\left(\mathrm{ddH}_{2} \mathrm{O}\right)$. The brains for all the conditions were then immersed for $30 \mathrm{~min}$ in equilibration buffer and incubated $1 \mathrm{~h}$ at $37^{\circ} \mathrm{C}$ with a solution containing $44 \mu \mathrm{l}$ of equilibration buffer, $5 \mu \mathrm{l}$ of marked nucleotide mixture, and $1 \mu \mathrm{l}$ of TdT enzyme (Promega). The reaction was stopped for $15 \mathrm{~min}$ in $2 \times$ SSC solution (diluted in $\mathrm{ddH}_{2} \mathrm{O}$ ) and washed three times in PBS. The brains were then mounted in Vectashield (Vector Laboratories).

RNA extraction. RNA was extracted as described previously (Napoletano et al., 2011). Briefly, 50 heads were cut and placed into a $1.5 \mathrm{ml}$ microfuge tube followed by a snap freeze in liquid nitrogen. The heads were then homogenized in $100 \mu \mathrm{l}$ of TriZOL (Invitrogen), after which an additional $200 \mu \mathrm{l}$ of TriZOL was added. The mixture was incubated for $10 \mathrm{~min}$ at RT before adding $60 \mu \mathrm{l}$ of chloroform. The tubes were vigorously mixed for $20 \mathrm{~s}$ and then incubated $2 \mathrm{~min}$ at RT before $15 \mathrm{~min}$ of centrifugation at $4^{\circ} \mathrm{C}$ and $12,000 \mathrm{rpm}$. Next, $200 \mu \mathrm{l}$ of the aqueous phase (containing the RNA) was transferred into another $1.5 \mathrm{ml}$ microfuge tube and $150 \mu \mathrm{l}$ of isopropanol was added. A pellet of RNA appeared after $10 \mathrm{~min}$ of centrifugation at $4^{\circ} \mathrm{C}$ and $12,000 \mathrm{rpm}$. The pellet was washed three times with $75 \% \mathrm{EtOH}$ and air-dried before the addition of $40 \mu \mathrm{l}$ RNase-free $\mathrm{ddH}_{2} \mathrm{O}$ with $1 \mu \mathrm{l}$ of Rnasin (Promega). The quality of the
RNA extraction was assessed on a $1.5 \%$ agarose gel and the concentration and $\lambda 280 / 260$ and $\lambda 260 / 230$ ratios were measured with a Nanodrop.

$q P C R$. A $1.5 \mu \mathrm{g}$ sample of each RNA was sent to the King's College London Genomic Centre Facility for processing. qPCR was done using Universal Probe Library (UPL) probes and primers designed by the Universal Probe Library software (Roche) for quantification. Three independent biological replicates were measured for each genotype at each time point. Gapdh and eIF4A were used as controls. Details of oligos and UPL are given in Table 1.

Western blot. An equal number of flies per genotype was decapitated with a scalpel, snap-frozen, and homogenized using a pestle in $1 \times$ sample buffer $(0.05 \mathrm{~mm}$ Tris- $\mathrm{HCl}$ pH 6.8, 2.5\% SDS, 10\% glycerol, $0.0025 \%$ bromophenol blue) with $5 \% \beta$-mercaptoethanol freshly added. The tube was centrifuged at $12,000 \mathrm{rpm}$ at $4^{\circ} \mathrm{C}$ for $5 \mathrm{~min}$. The supernatant was transferred into a new tube. The volume equivalent to five heads was loaded on $10 \%$ or $12 \% \mathrm{w} / \mathrm{v}$ polyacrylamide gel using a Bio-Rad gel electrophoresis apparatus. After separation in the gel, proteins were transferred onto a nitrocellulose blotting membrane $(0.2 \mu \mathrm{m}$, Protran; GE Healthcare) for $1 \mathrm{~h}$ at $60 \mathrm{~V}$ and kept cold. The membrane was then blocked for $1 \mathrm{~h}$ in 5\% BSA or 5\% milk in TBS with Tween (TBS-T) at RT before incubation in primary antibody diluted in blocking buffer overnight at $4^{\circ} \mathrm{C}$. The membrane was washed 3 times for $15 \mathrm{~min}$ in TBS-T at RT and then incubated $1 \mathrm{~h}$ at RT in secondary antibody. After three washes in TBS-T, the membrane was washed again in TBS. Enhanced chemiluminescence (ECL) reagent was mixed according to the manufacturer's instructions (SuperSignal WestPico or ECL Western blotting substrate; Pierce) and spread onto the membrane. After 2 min of incubation, the ECL liquid was removed and the membrane placed into a cassette with a film (Fujifilm) and developed. The film 
A $3 \mathrm{~d} @ 29^{\circ} \mathrm{C} \rightarrow 18^{\circ} \mathrm{C}$

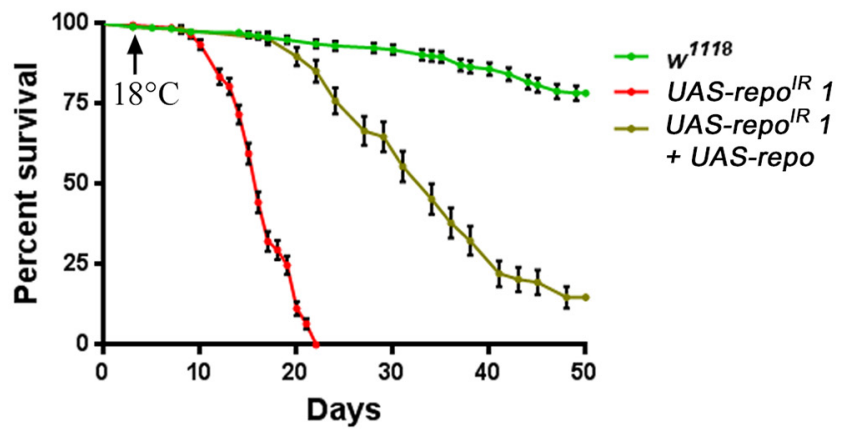

C

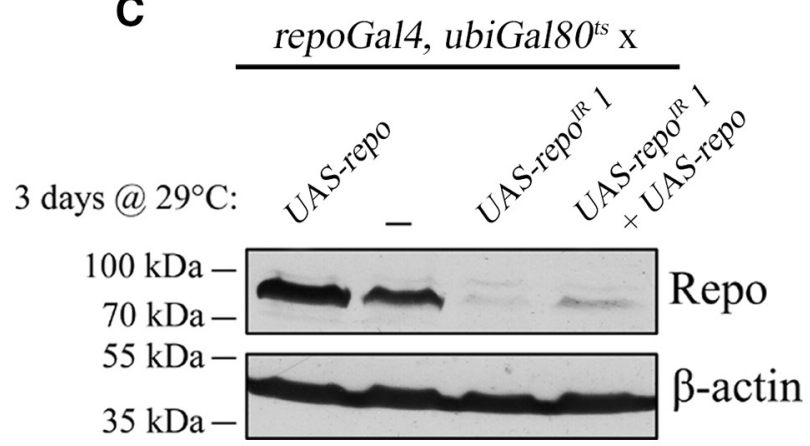

E

$3 \mathrm{~d} @ 29^{\circ} \mathrm{C}+20 \mathrm{~d} @ 18^{\circ} \mathrm{C}$

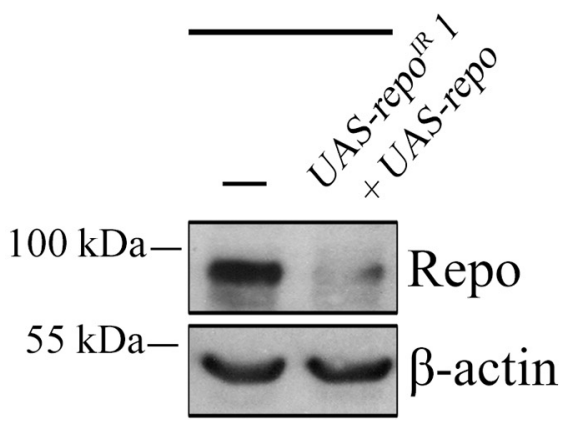

B

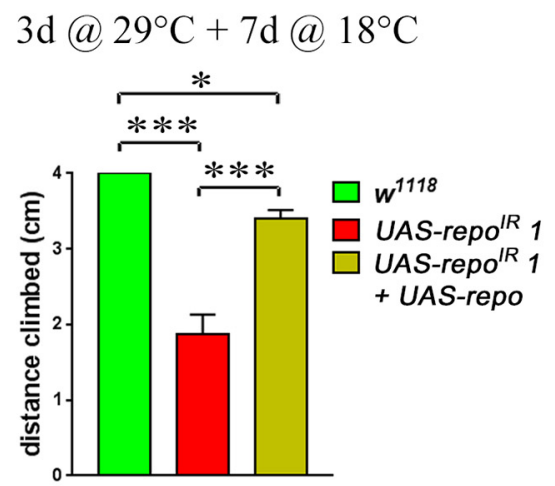

D

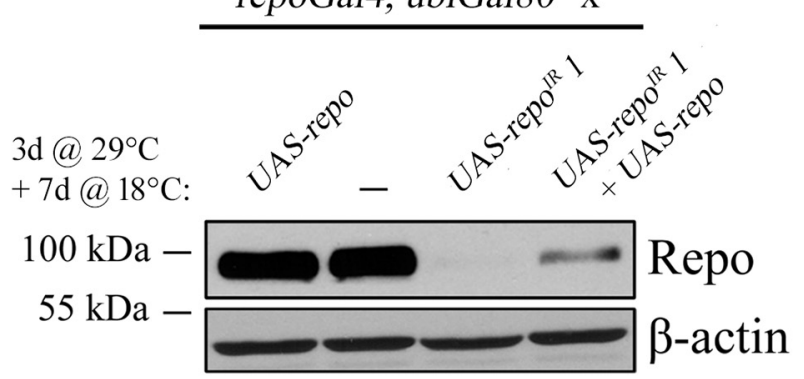

$\mathbf{F}$ repoGal4, ubiGal80 $\mathrm{x}$

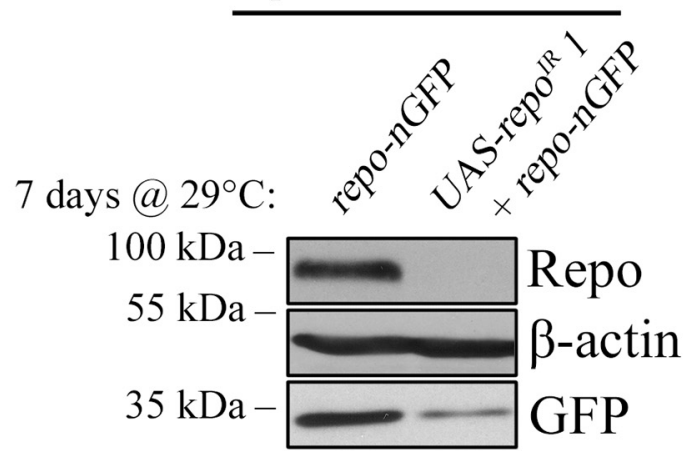

Figure 3. repo expression is continuously required. $A$, Lifespan of repo $0^{\mathbb{R}} 1$ flies and their rescue after $3 \mathrm{~d}$ of transgene expression at $29^{\circ} \mathrm{C}$ followed by a block of transgene expression with a transfer at $18^{\circ} \mathrm{C}$ (indicated by an arrow on the graph). B, Climbing assay of the same genotypes as in $\boldsymbol{A}$ using the same paradigm. The assay was done after $7 \mathrm{~d}$ at $18^{\circ} \mathrm{C}$, prior to death of the repo $0^{\mathrm{R}} 7 \mathrm{flies}$ (one-way ANOVA, Fisher's $L S D p=0.0001, p=0.0448$ and $p=0.0007$, respectively). $\mathbf{C} \boldsymbol{E}$, Western blot analysis of Repo expression after $3 \mathrm{~d}$ at $29^{\circ} \mathrm{C}(\boldsymbol{C})$. Note the evident overexpression of Repo from an exogenous transgene, which partially counteracts Repo knock-down by the RNAi, after a further $7 \mathrm{~d}$ at $18^{\circ} \mathrm{C}(\boldsymbol{D})$, representing the levels of Repo at the same time point as the climbing assay. Note the absence of Repo overexpression by the UAS-repo transgene compared with controls. After $3 \mathrm{~d}$ at $29^{\circ} \mathrm{C}$ and 20 more days spent at $18^{\circ} \mathrm{C}(\boldsymbol{E})$, the Repo levels in UAS-repo ${ }^{\mathbb{R}} 1+U A S$-repo condition remains very low even after a long period of transgene expression blockage. The driver used was ubiGal $80^{\text {ts }} ;$ repoGal4.F, Analysis of nGFP protein levels from the repo-nGFP transgene after $7 \mathrm{~d}$ of UAS-repo $0^{\mathbb{R}} 1$ expression. The driver used was ubiGal $80^{\text {ts }}$; repoGal4. ${ }^{*} p<0.05,{ }^{* * *} p<0.001$.

was then scanned and processed using Adobe Photoshop version 7.0.1 (RRID:SCR_014199) and the bands were quantified using Image Studio Lite version 4.0 software (RRID:SCR_013715). Antibodies were used as follows: anti-Repo (mouse, $1 / 200$ in 5\% BSA, DSHB 8D12, RRID:AB_528448), anti-Gat (rabbit, $1 / 10000$ in 5\% milk-TBS-T, RRID:AB_2569706, gift from Marc Freeman), anti- $\beta$-actin (rabbit, $1 / 3000$ in $5 \%$ BSA, SAB 21338), anti-myc (mouse, $1 / 1000$ in 5\% BSA, Roche 9E10, RRID:AB_439694), and anti-GFP (mouse, 1/1000 in 5\% BSA, Roche, RRID:AB_390913).

Statistical analysis. All statistical analysis was performed with GraphPad Prism software (RRID:SCR_002798). For all lifespans, the statistical analysis was performed using the log-rank test of the Kaplan and Meier method. For the climbing assay in Figure $2 B$ and behavioral experiments (DART), the statistical analysis was done by one-way ANOVA using Dunnett's multiple-comparisons post hoc test. For the analysis of experiments performed over time (climbing of Fig. $6 A, F, H$; seizure in Fig. $8 B$; and behavior in Fig. $8 A$ ) a two-way ANOVA was used with a Tukey's or Dunnett's multiple-comparisons test. All quantifications for Western blots and climbing assay with only two genotypes were analyzed at high stringency using a nonparametric Mann-Whitney test. The qPCR statistical analysis was performed using DataAssist software (RRID:SCR_014969) as a two-tailed $t$ test. Significance is shown by asterisks in all figures as follows: ${ }^{\star} p<0.05,{ }^{* *} p<$ $0.01,{ }^{* * *} p<0.001$, and ${ }^{* * *} p<0.0001$.

\section{Results}

miR overexpression screen in adult glial cells

Using the repo Gal4, ubiGal80 ${ }^{\text {ts }}$ inducible system of expression, we have triggered expression in all adult fly glia of several Drosophila miRNAs from a library of $>250$ lines (Bejarano et al., 2012) and 
A

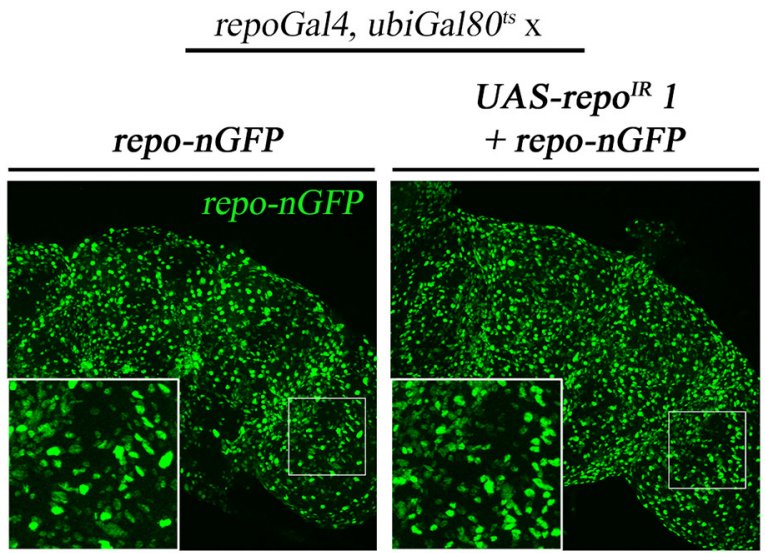

B

repoGal4, ubiGal80 $0^{\text {ts }} \mathrm{x}$

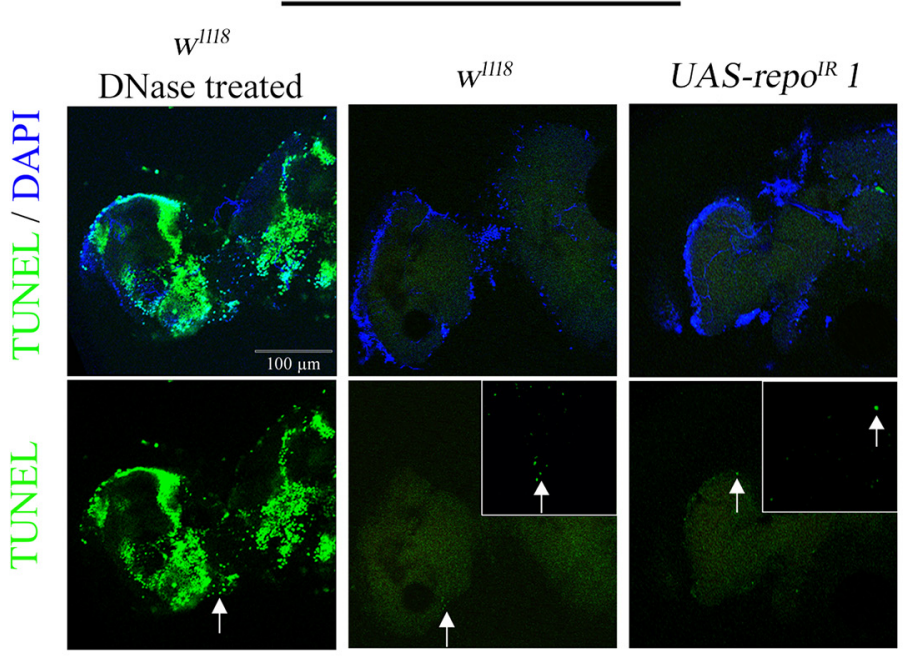

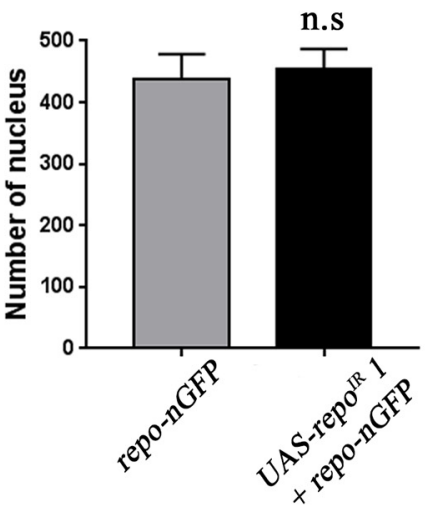

C
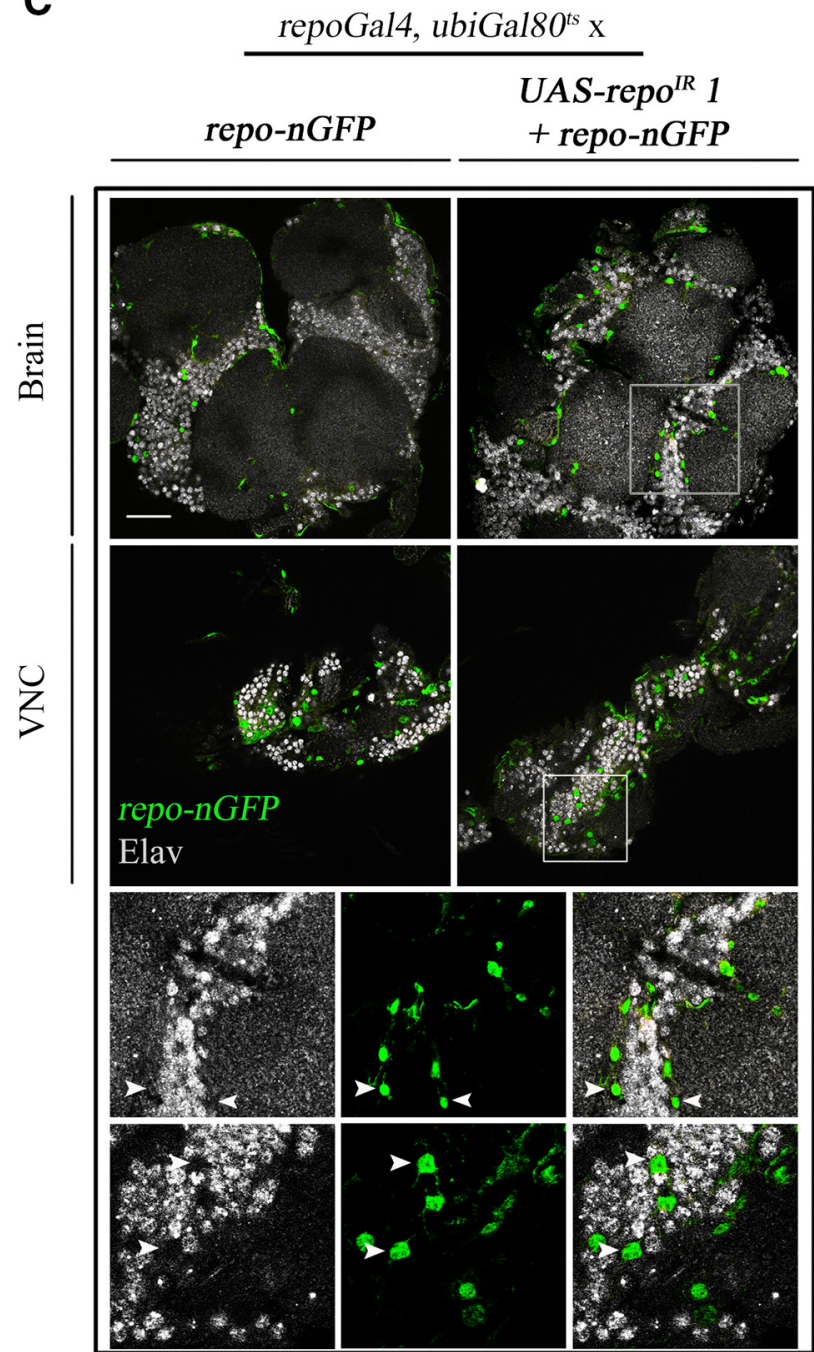

Figure 4. Repo knock-down in the adult does not trigger cell death or change of fate. A, Maximum projections of confocal images of $w^{1118}$ and UAS-repo ${ }^{1 R} 1$ brains after $7 \mathrm{~d}$ at $29^{\circ} \mathrm{C}$. Glial cells were labeled in green using the repo-nGFP transgene used for previous Western blot (Fig. 3F). Quantification was done in the optic lobes of three independent brains for each genotype. No significant difference was observed between control and RNAi conditions. B, TUNEL assay using the same genotypes and the same conditions as previously. A positive control for the assay was done by treating control brains with DNase prior to labeling. No difference could be observed between control and RNAi conditions. See Materials and Methods for detailed protocol. C, Immunostaining of the brain and VNC of $w^{1118}$ and UAS-repo ${ }^{\mathbb{R}} 1$ flies after $7 \mathrm{~d}$ at $29^{\circ} \mathrm{C}$. In green is nGFP from the repo-nGFP transgene and in gray is the pan-neuronal marker Elav. The images represent confocal sections at the level of the antennal lobes for the brains and abdominal segment for the Ventral Nerve Cord (VNC). Bottom panels represent higher magnification of the UAS-repo ${ }^{\mathbb{R}} 1$ condition in the brain (top) and in the VNC (bottom). Arrowheads indicate presumptive glial cell nucleus. There was no overlap between GFP and Elav staining in either case. The driver used was $u b i G a / 80^{\text {ts }}$; repoGal4. Scale bar, $30 \mu \mathrm{m}$. 
A

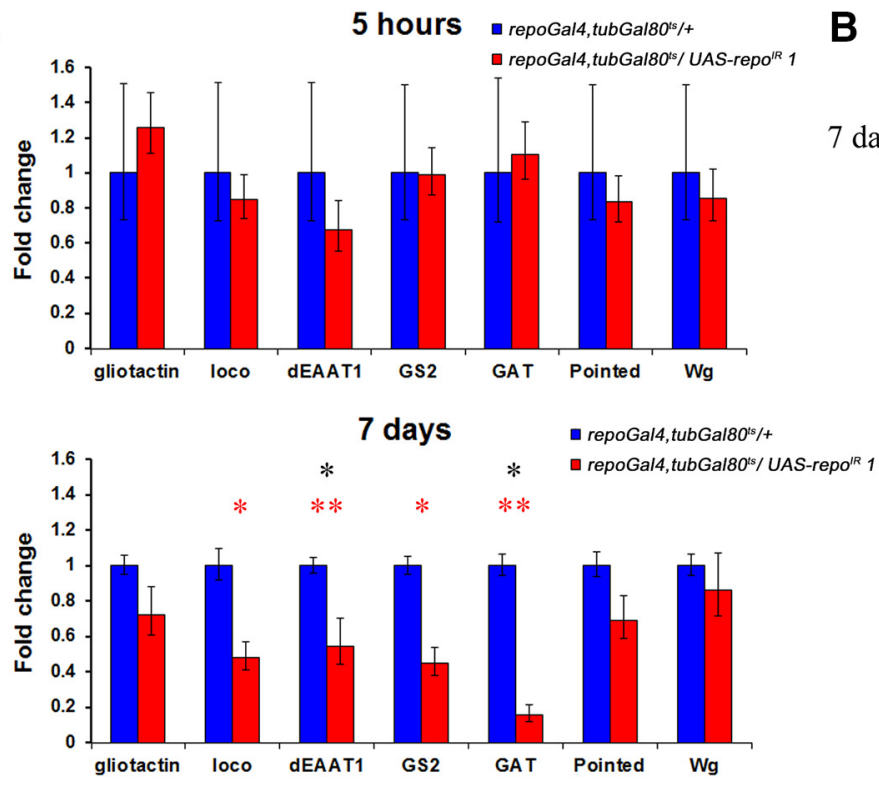

B
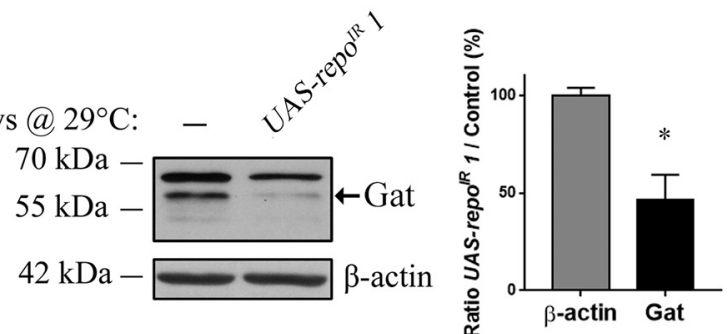

C

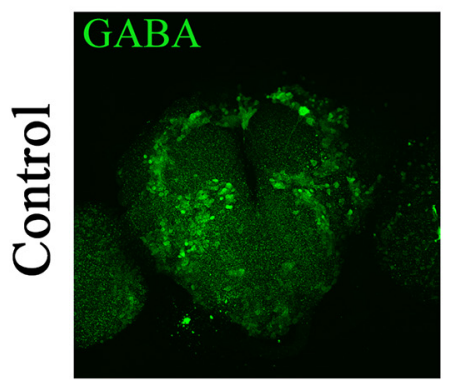

GABA intensity in UAS-repo ${ }^{\mathbb{R}} 1$ flies
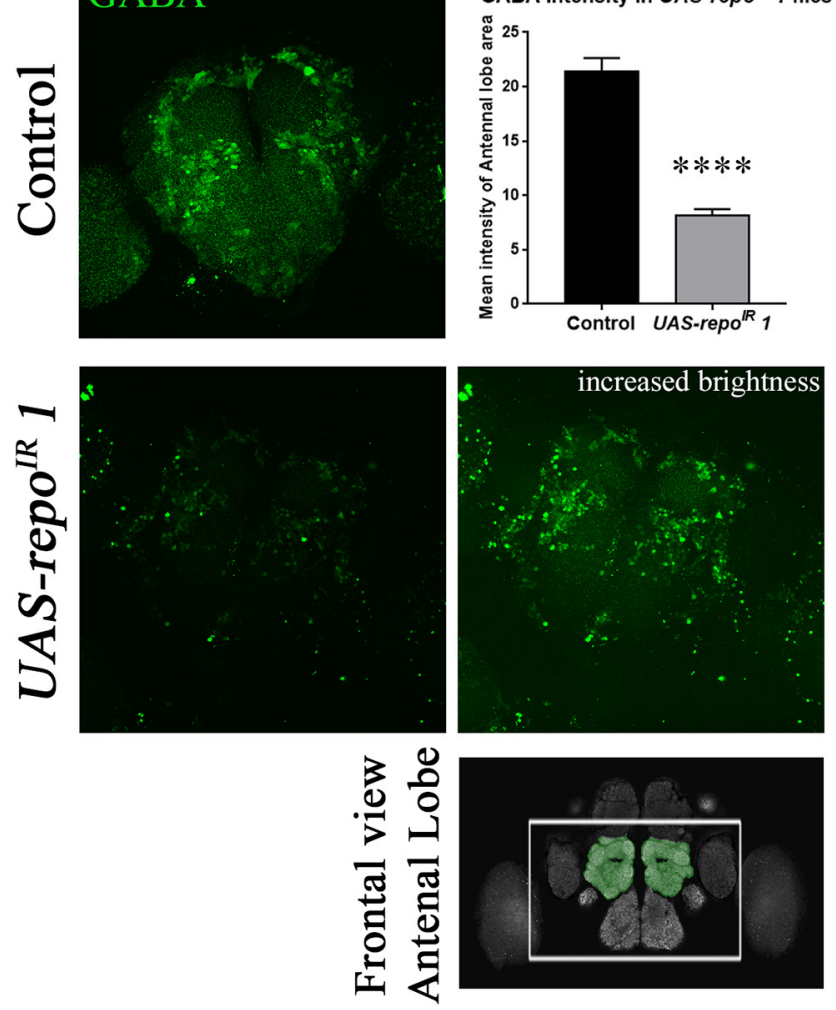

D
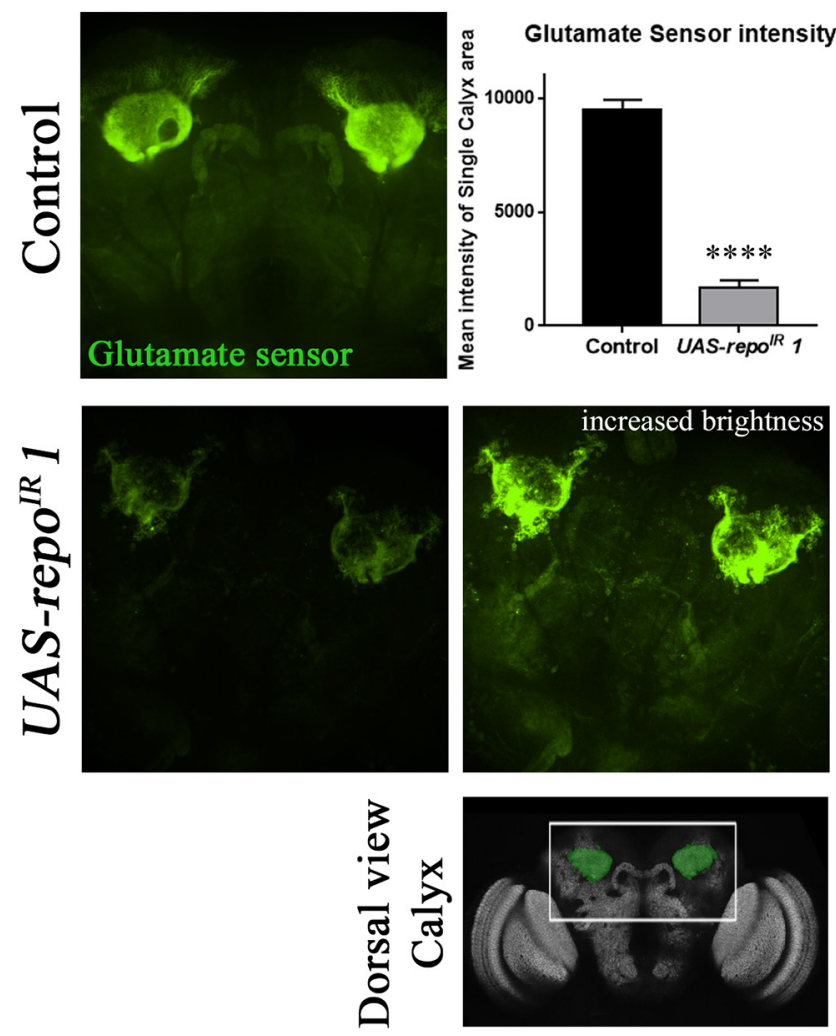

Figure 5. Analysis of Repo targets and effect on GABA and glutamate levels. All aging in this figure was performed at $29^{\circ} \mathrm{C} . \mathrm{A}, \mathrm{qPCR}$ analysis of known Repo transcriptional target genes and of Gat after $5 \mathrm{~h}$ or $7 \mathrm{~d}$ at $29^{\circ} \mathrm{C}$ in $w^{1118}$; tubGal80 ${ }^{\text {ts }}$, repoGal4/+ (control) and $w^{1118} ;$ UAS-repo ${ }^{\mathbb{R}} 1 /+;$ tubGal80 ${ }^{\text {ts }}$, repoGal4/+ flies. Each condition was measured with three independent biological replicates. No statistically significant difference was observed between control and the expression of UAS-repo ${ }^{\mathbb{R}} 7$ after $5 \mathrm{~h}$ at $29^{\circ} \mathrm{C}$ (before the RNAi can downregulate repo). After $7 \mathrm{~d}$, Ioco, dEAAT1, Gs2, and Gat were significantly downregulated at the mRNA level. Asterisks represent significance before [red, two samples, two-tailed $t$ test, $n=3$ from left to right $p=0.0289$ (loco), $p=0.0013$ (dEAAT1), $p=0.0197$ (GS2), $p=0.0029$ (Gat)] and after [black, $p=0.013$ (dEAAT1), $p=0.0143$ (Gat)] false discovery rate (FDR) correction. $\boldsymbol{B}$, Western blot analysis of Gat expression and its quantification. The expected molecular weight of Gat is $57 \mathrm{kDa}$. The quantification was done with four independent biological replicates. Mann-Whitney, $n=4, p=0.0143$. C, Immunostaining using a specific anti-GABA antibody (green) of Drosophila brains after $7 \mathrm{~d}$ at $29^{\circ} \mathrm{C}$. The images represent the maximum projection of $z$-stacks of the central brain taken with a $40 \times$ oil-immersion objective. The quantification is the average of the pixel intensity given for the area around each antennal lobe for three brains per genotype (unpaired $t$ test, two-tailed, $p<0.0001$ ). The measurements were done using ImageJ Fiji software. The image of a Repo-RNAi brain with a higher brightness (brightness parameter brought from 255 to 80 ) shows the presence of the cells and their localization, barely visible under the conditions used for the control brains. A schematic of the brain region observed is highlighted below. $\boldsymbol{D}$, Fluorescence intensity generated in the adult brain by the glutamate sensor UAS-iGluSnFRA184S expressed alone or in combination with UAS-repo ${ }^{\mathbb{R}} 1$ after $7 \mathrm{~d}$ at $29^{\circ} \mathrm{C}$. The images were taken in the brain area highlighted (Figure legend continues.) 
recovered a number of miRNAs that shorten or extend the fly lifespan compared with the screen median lifespan (Fig. 1A,B and Fig. 1-1, available at https://doi.org/10.1523/JNEUROSCI. 1833-18.2019.f1-1).

Although not isogenic, the library was generated in the $w^{1118}$ background. We therefore ran a number of additional controls by crossing repoGal4,ubiGal80 ${ }^{\text {ts }}$ to $w^{1118}$ throughout the screen to compare their F1 with the median lifespan of our screen. Given the significant difference (Fig. 1C), we decided to focus on miRNAs that shorten lifespan because this class appears more robust compared with both controls.

\section{miR-1 expression in adult glia shortens the fly lifespan by repressing the expression of repo}

Two independent UAS-miR-1 insertions displayed a potent reduction in fly lifespan when expressed in the adult glia (Fig. $1 A, D$ and Fig. 1-1, available at https://doi.org/10.1523/JNEUROSCI. 1833-18.2019.f1-1). miR-1 is known to be expressed in the mesoderm during embryogenesis (Sokol and Ambros, 2005) and has been involved in setting up the repression of nonmuscle cell genes (Lim et al., 2005). In analyzing the predicted mRNA targets for miR-1 in the TargetScan and microRNA.org databases, we noticed that the pan-glial marker repo was predicted to be a target for miR-1. In agreement, specific adult expression of miR-1 in glia leads to a strong downregulation of the Repo protein expression in adult flies (Fig. 1E). We have reported previously that this effect is likely to be direct (Trébuchet et al., 2019). An exogenous UAS-repo-myc lacking the repo 3 '-untranslated region and therefore resistant to repression by miR-1 (data not shown) significantly rescues the effect of miR-1 on lifespan (Fig. 1F). Conversely, a UAS-repo $\Delta H D-m y c$ lacking the homeodomain failed to rescue the miR-1 lifespan phenotype (Fig. 1F), highlighting the requirement for a functional Repo protein and ruling out that the rescue by UAS-repo may be nonspecific due to the addition of a second UAS transgene.

\section{Repo is continuously required in adult glial cells for fly survival and motor behavior}

The regulation of Repo by miR-1 is physiologically relevant during the developmental stages for hemocyte and glial cell specification (Trébuchet et al., 2019). Here, we used miR-1 ectopically as a discovery tool in the adult. Our results suggest that Repo may be specifically required in adult glial cells. It was previously shown that some repo alleles lead to neurodegeneration (Xiong and Montell, 1995); however, the use of genetic mutations does not allow us to exclude that neurodegeneration arises as a secondary consequence of developmental abnormalities. The inducible system of expression that we use here allows us instead to specifically investigate functions in adult nervous system maintenance. Adult-specific repo RNAi led to dramatic shortening of the fly lifespan (Fig. 2A) and progressive loss of motor activity (Fig. 2B). Both phenotypes were partially rescued by an exogenous UASrepo (Fig. $2 A, B$ ), which had no effect per se (data not shown). This transgene is still sensitive to the RNAi effect, probably

\section{$\leftarrow$}

(Figure legend continued.) in the schematic below the pictures. The settings of the microscope were adjusted with the first control brain analyzed and kept unmodified. Quantifications were done using ImageJ Fiji software, drawing a square around each calyx region, measuring the intensity of the brightest slices of the stack, and then averaging (unpaired $t$ test, two-tailed, $p<0.0001$ ). The genotypes used were as follows: elav-Gal4; tubGal80 ${ }^{\text {ts }}$, repoGal4/UAS-iGluSnFRA184S as control and elav-Gal4; UAS-repo ${ }^{\text {IR }} 1 /+$; tubGal80 ${ }^{\text {ts }}$, repoGal4/UAS-iGluSnFRA184S. explaining the mild degree of phenotypic rescue and of Repo protein expression achieved (Fig. 2C). Downregulating repo in different subsets of glial cells using other glial Gal4 drivers (astrocyte-like/alrmGal4, cortex/NP2222Gal4, ensheathing/ MZ0709Gal4, and subperineural/moodyGal4 glia) did not markedly affect lifespan (Fig. 2D). Only dEAAT1Gal4 (expressed in astrocyte-like glia, cortex glia, and some subperineurial glia; data not shown) and the ubiquitous Actin5cGal4 elicited a more robust phenotype in this assay, suggesting that this phenotype results from a combination of factors rather than one single function in a specific glia subset.

We next tested whether repo expression is continuously required in the adult fly. At $18^{\circ} \mathrm{C}$, repo RNAi and an exogenous UAS-repo transgene are efficiently repressed by $u b i G a l s 0^{\text {ts }}$ (data not shown). We have induced repo RNAi for the first $3 \mathrm{~d}$ of adult life only at $29^{\circ} \mathrm{C}$, followed by phenotypic analysis back at $18^{\circ} \mathrm{C}$, when transgenes are no longer transcribed. The effects of just $3 \mathrm{~d}$ at $29^{\circ} \mathrm{C}$ on lifespan and motor activity were dramatic (Fig. $3 \mathrm{~A}, \mathrm{~B}$ ) and in both cases could be partially rescued by an exogenous UAS-repo transgene. At the protein level, $3 \mathrm{~d}$ at $29^{\circ} \mathrm{C}$ led to an efficient knock-down (Fig. $3 C$ ), but there was no recovery back at $18^{\circ} \mathrm{C}$ (Fig. $3 D, E$ ). The dramatic effect of a temporary knockdown is therefore likely due to the requirement for the Repo protein to maintain repo gene expression. Accordingly, a transgenic repo-nGFP was also dramatically reduced by the repo RNAi (Fig. $3 F$ ), confirming the presence of a regulatory sequence within the $4.5 \mathrm{~kb}$ repo promoter (Lee and Jones, 2005; Flici et al., 2014).

\section{Expression of genes involved in GABA and glutamate recycling requires Repo in adult glia}

The data collected so far indicated a continuous requirement for the Repo protein in adult glia to maintain its own expression, which in turn supports fly lifespan, endogenous locomotor activity, and response behavior to an exogenous mechanical stimulation.

Investigating the possible biological cause for the dramatic phenotype in the repo-KD flies, we did not find any evidence for glial cell loss (Fig. 4A), apoptotic cell death (Fig. 4B), or fate switch of glia to neurons (Fig. 4C), all of which take place during development (Trébuchet et al., 2019). We also did not detect gross morphological alterations to the shape of glial cells and of the major nerve fiber tracts (data not shown).

To obtain an indication of the processes that could be disrupted in repo-KD flies, we monitored by qPCR the expression of several genes (Gliotactin, loco, dEAAT1, Gs2, pointed, and wingless), recently established as direct targets of Repo (Kerr et al., 2014). loco and Gliotactin are genes involved in the signaling pathway setting up the fly "blood-brain barrier" (Auld et al., 1995; Schwabe et al., 2005). pointed and wingless are important for glial specification and development (Klaes et al., 1994; Kerr et al., 2014). In addition, we have examined the expression of the only Drosophila glial GABA transporter gene, Gat, to complement, together with $d E A A T 1$ and Gs2, the key components of the glutamate/GABA/glutamine cycle (Roth and Draguhn, 2012; Rowley et al., 2012). After 7 d, loco, Gs2, Gat, and dEAAT1 mRNA levels were significantly downregulated (Fig. 5A). Using a specific antibody (Stork et al., 2014), we have confirmed the downregulation of Gat also at the protein level (Fig. 5B).

Interestingly, the downregulation of Gat, $d E A A T 1$, and Gs2 suggested that the glutamate/GABA/glutamine cycle may be severely compromised in repo-KD flies. This cycle in glia has been shown to be of major importance in the maintenance of the glutamate and GABA pool in neurons (Rae et al., 2003). Indeed, antibody 
A Negative Geotaxis Response

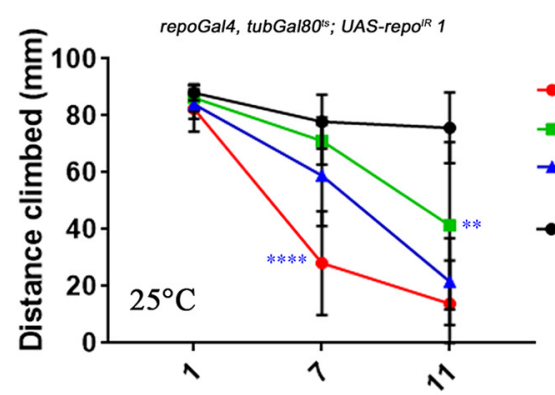

Days
B

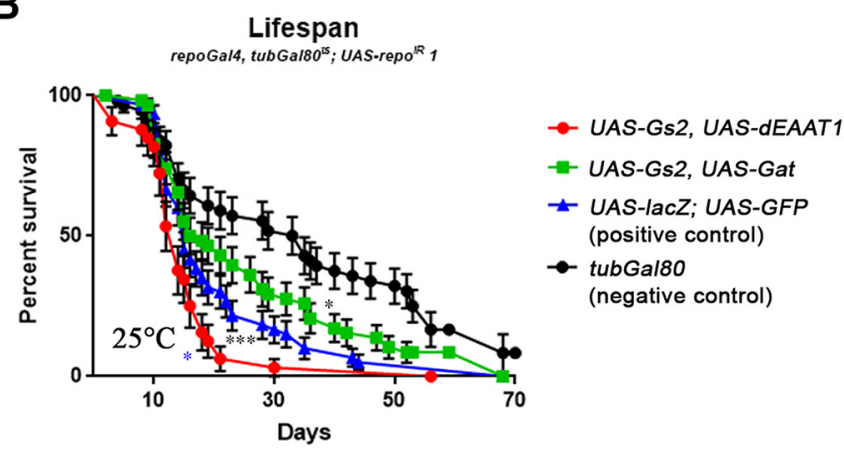

C

repoGal4, tubGal80 $0^{t s}$ UAS-repo ${ }^{I R} 1 \mathrm{x}$

17 days @ $25^{\circ} \mathrm{C}$ :

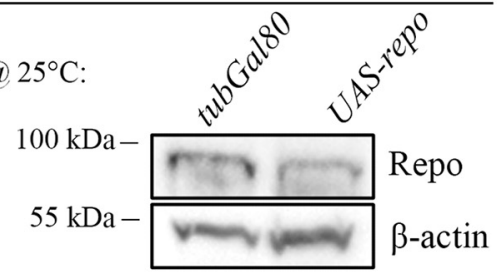

D

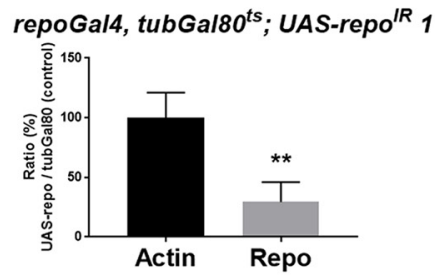

E

repoGal4,tubGal80 $0^{t s}$; UAS-repo ${ }^{I R} 1 \mathrm{X}$

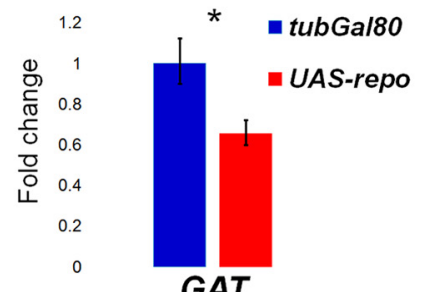

F Negative Geotaxis Response

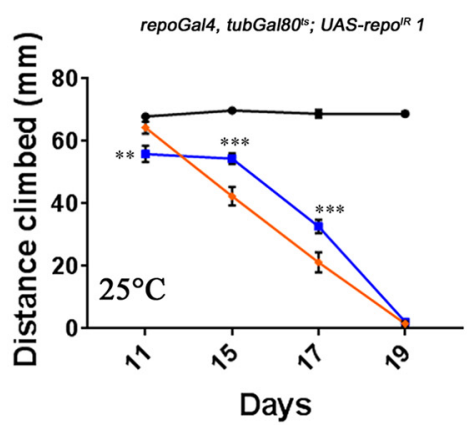

H

Negative Geotaxis Response

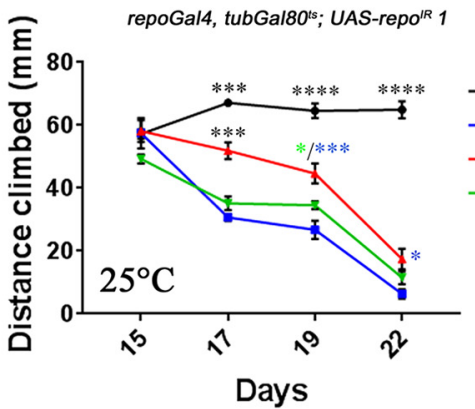

$\rightarrow$ tubGal80 (control)

- UAS-repo

$\rightarrow$ UAS-dEAAT1, UAS-repo

- tubGal80 (control)

- UAS-repo

- UAS-Gat; UAS-repo

- UAS-Gs2; UAS-repo
G

Lifespan

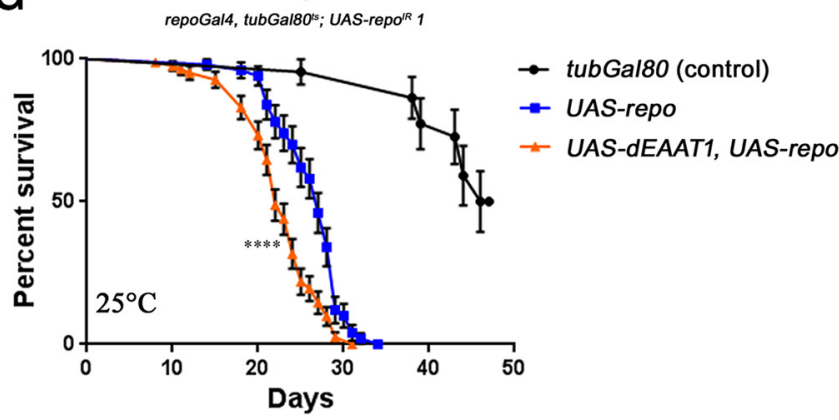

I

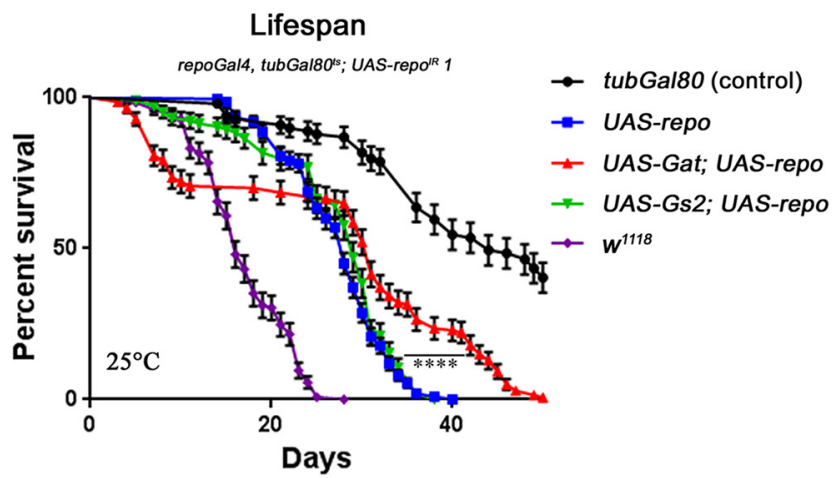

Figure 6. Effect of Gat, GS2 and dEAAT1 levels on behavioral and lifespan deficits caused by repo knock-down. All rescue experiments in this figure and in the related Figures 7 and 8 , were performed at $25^{\circ} \mathrm{C}$ and genotypes used were $w^{1118} ;$ UAS-repo ${ }^{\mathbb{R}} 1 /+;$ tubGal $80^{\text {ts }}$, repoGal4/tubGal80 (negative control) // $w^{1118} ;$ UAS-repo $0^{\mathbb{R}} 1 /$ UAS-lacZ; tubGal80 ${ }^{\text {ts }}$, repoGal4/UAS-eGFP $/ / w^{1118}$; UAS-repo ${ }^{\mathbb{R}} 1 /$ UAS-GS2; tubGal80 ${ }^{\text {ts }}$, repoGal4/UAS-dEAAT1 // $w^{1118}$; UAS-repo ${ }^{\mathbb{R}} 1 /$ UAS-GS2,UAS-Gat; tubGal80 ${ }^{\text {ts }}$, repoGal4/+ $/ / w^{1118}$; UAS-repo ${ }^{\mathbb{R}} 1 /$ UASeGFP; tubGal80 $^{\text {ts }}$, repoGal4/UAS-repo $/ /$

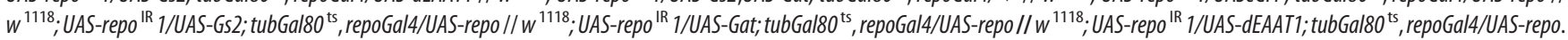
$A$, Evolution over time of the negative geotaxis response. Ten to 15 flies were assessed three times at different time points at $25^{\circ} \mathrm{C}$. Coexpression of UAS-Gs2 and UAS-Gat delays the decrease in climbing ability over time compared with the positive control, whereas coexpression of UAS-Gs2 and UAS-dEAAT1 transiently aggravates the phenotype. Blue asterisks indicate significance compared with the positive control (two-way ANOVA, Tukey's multiple-comparisons test $p<0.0001$ and $p=0.0011$, respectively). $\boldsymbol{B}$, Fly lifespan was assessed with control flies carrying the tubGal80 transgene (not temperature sensitive) to block the Gal4 activity. The positive control flies expressing control proteins in a repo-KD background display a significantly shorter lifespan compared with the negative control. Coexpression of UAS-Gs2 and UAS-dEAAT1 further shortens the lifespans of repo-KD flies, whereas coexpression of UAS-Gs2 and UAS-Gat displays (Figure legend continues.) 
staining for the GABA neurotransmitter revealed a striking reduction in GABA levels in neurons (Fig. 5C). A similar effect was observed for glutamate as monitored by the iGLUSnFRA184S glutamate sensor (Marvin et al., 2013; Stork et al., 2014). The iGLUSnFRA184S signal was stronger in the calyx and mushroom body regions, which are known to have high levels of glutamate (Daniels et al., 2008; Sinakevitch et al., 2010), and was dramatically decreased in repo-KD flies (Fig. 5D).

Therefore, repo expression in the adult glia is necessary for the maintenance of neurotransmitter homeostasis, likely via the transcriptional regulation of components of the glutamate/ GABA/glutamine cycle.

\section{Gat overexpression in adult glia delays the emergence of motor dysfunction and decreases the sensibility to seizures}

Rescue experiments to analyze the causal role of the downregulation of genes controlling the glutamate/GABA/glutamine cycle for the repo-KD phenotypes were performed at $25^{\circ} \mathrm{C}$ instead of $29^{\circ} \mathrm{C}$ to allow slower progression of defects. As negative control for the phenotypes analyzed, we have used tubGal80 to inhibit expression of all transgenes while maintaining all common genomic elements than all other stocks analyzed (repoGal4, tubGalso $0^{\text {ts }}$, and UAS-repo ${ }^{\mathrm{IR}} 1$ ). The positive controls were repo-KD flies expressing control proteins LacZ and GFP. These flies were not included in all assays because they become immobile or die too early for meaningful comparison at the late stages. Gal4/UAS mediated overexpression of only one of the genes tested (Gat, Gs2, and dEAAT1) was unable to rescue either motor defects or lifespan in repo-KD flies (data not shown). However, a mild but significant rescue was detected upon Gs2 and Gat, but not $G s 2$ and $d E A A T 1$ co-overexpression in climbing assays (Fig. $6 A$ ). The coexpression of Gs 2 and $d E A A T 1$ was rather transiently detrimental in this assay and in lifespan analysis (Fig. 6B). At the level of lifespan, the coexpression of $G s 2$ and Gat only displayed a trend toward rescue, but was not significantly different from the

\footnotetext{
$\leftarrow$

(Figure legend continued.) a trend toward rescue but no significant difference. Black asterisks indicate significance compared with the negative control; blue asterisks indicate significance against the positive control (log-rank Mantel-Cox test $p=0.0207, p=0.0001$ and $p=$ 0.0134 , respectively). $C$, Western blot showing the downregulation of Repo protein in rescuedrepo-KD compared with control flies. Actin is used as a loading control. D, Quantification of Repo protein expression. Mann-Whitney, $n=6, p=0.0022$. E, UPL qPCR assay for Gat (as performed in Fig. 5A). Values are normalized using elF4A as control. Unpaired $t$ test, two-tailed, $n=3 p=0.024$. $F$, Evolution over time of the negative geotaxis response as in $\boldsymbol{A}$. Coexpression of UAS-dEAAT1 and UAS-repo accelerates the decrease in climbing ability over time. Black asterisks indicate significance compared with the negative control (two-way ANOVA, Tukey's multiple-comparisons test, $p=0.0068, p=0.0001$, and $p=0.0003$, respectively). G, Fly lifespan was assessed as in $\boldsymbol{B}$. Similar to the climbing assay, the coexpression of UAS-dEAAT1 beside UAS-repo does not rescue and rather aggravates the lifespan phenotype. Asterisks indicate significance compared with UAS-repo alone (log-rank Mantel-Cox test $p=$ $0.000010383935)$. $\boldsymbol{H}$, Evolution over time of the negative geotaxis response as in $\boldsymbol{A}$. The flies coexpressing UAS-Gat and UAS-repo display a significantly improved climbing abilities compared with UAS-repo alone or UAS-repo and UAS-Gs2. Black asterisks indicate significance in genotype/time interactions compared with UAS-repo alone in two-way ANOVA (Tukey's multiple-comparisons test $p<0.0001, p=0.0009, p<0.0001$, and $p<0.0001$, respectively). Colored asterisks indicate significance between the different genotypes and UAS-repo alone at a specific time point (main genotype factor in two-way ANOVA, Tukey's multiplecomparisons test $p=0.0408, p=0.0001$ and $p=0.0207$, respectively). I, Conditions of the lifespan similar to $G$, but with the addition repo-KD flies as a positive control genotype ( $w^{1118}$; UAS-repo $^{\mathbb{R}} 1 /+$; tubGal $80^{\text {ts }}$, repoGal4/+). The coexpression of UAS-GS2 does not affect the lifespan, whereas UAS-Gat mildly but significantly increases the lifespan of $70 \%$ of the flies. Surprisingly, $\sim 30 \%$ of the flies in this condition die within $10 \mathrm{~d}$, whereas $25-30 \%$ of the population have a strong increase in lifespan. Asterisks indicate significance compared with UAS-repo alone (log-rank Mantel-Cox test $p=0.000000018838$ ).
}

positive control (Fig. 6B). To determine the individual contribution of single genes involved in the glutamate/GABA/glutamine cycle, we expressed them separately in synergy with an exogenous repo transgene in repoGal4, ubiGalso ${ }^{\text {ts }}>U A S-$ repo ${ }^{\mathrm{IR}}$, UAS-repo (hereafter rescued-repo-KD) flies. When aged at $25^{\circ} \mathrm{C}$, the rescued-repo-KD flies still display a downregulation of Repo to $\sim 1 / 3$ of control levels (Fig. $6 C, D$ ) and a milder but significant downregulation of Gat expression at $\sim 2 / 3$ of control levels (Fig. $6 E)$. Therefore, this represents an ideal background in which to assess the effect of exogenous expression of each of the three components of the glutamate/GABA/glutamine cycle analyzed here. Coexpression of UAS-dEAAT1 in rescued-repo-KD flies rather resulted in a detrimental effect both in the negative geotaxis climbing assay (Fig. $6 F$ ) and in lifespan (Fig. 6G) similar to what observed in combination with Gs2 (Fig. $6 A, B$ ). In contrast, coexpression of UAS-Gat significantly improved the climbing ability in rescued-repo-KD flies (Fig. $6 H$ ). At the level of lifespan, a mild but significant rescue was detected (Fig. 6I), but resulted from a consistent biphasic effect. The reasons for the biphasic lifespan of rescued-repo-KD flies coexpressing UAS-Gat are unknown and were not present when Gat was expressed alone or in combination with Gs2 (Fig. 6B).

Given the pleiotropy of lifespan effects, we reasoned that a different assay was necessary to effectively determine all components involved in the regulation of the glutamate/GABA/glutamine cycle by Repo. Therefore, we focused our analysis on specific behavioral deficits in the repoGal4, ubiGalso ${ }^{\text {ts }}>U A S$ repo ${ }^{\mathrm{IR}}$ flies (hereafter repo-KD). For refined and unbiased investigations, we established a novel DART paradigm (Faville et al., 2015). This allows automated video-assisted motion tracking and stimuli response to mechanical shock to investigate endogenous and exogenous locomotor activity (Fig. $7 A$; see Materials and Methods for detailed protocol). After $7 \mathrm{~d}$ at $29^{\circ} \mathrm{C}$, whereas control flies (that carry all relevant transgenes, however repressed by tubGal80) respond to the series of vibrations by a sudden transient increase in speed (represented by a peak in relative speed), the repo-KD flies did not respond to the stimuli provided (Fig. $7 B$ ). This defect was also partially but significantly rescued by the expression of an exogenous UASrepo. Therefore, our novel DART setup is capable of robust and sensitive analysis of motor behavior.

Using our DART setup, we analyzed the effect of UAS-Gat in more detail. Indeed, whereas all tested genotypes are indistinguishable after $3 \mathrm{~d}$ at $25^{\circ} \mathrm{C}$, after $11 \mathrm{~d}$ at $25^{\circ} \mathrm{C}$ (a stage when climbing defects are not yet observed in rescued-repo-KD flies), this setup is able to detect a dramatic reduction in the response to the given stimuli. At this stage, coexpression of UAS-Gat significantly improves the amplitude of response to the level of the control flies, in which all transgenes are silenced and this improvement is still present after $17 \mathrm{~d}$ at $25^{\circ} \mathrm{C}$ (Fig. $7 \mathrm{C}$ ).

Surprisingly, the defects in stimuli response by rescuedrepo-KD flies do not arise because the flies are slower when active. Indeed, their speed while active was comparable to control unaffected flies at days 11 and 17 and, if anything, even faster at day 3 (Fig. 8A). Consistently, the rescue operated by UAS-Gat was not due to an increase of this parameter.

Interestingly, when analyzing the overall activity of these flies for the whole $2 \mathrm{~h}$ of the assay paradigm, not limited therefore to the sole response to stimulus and more representative of endogenously generated motor activity, it is apparent that the rescuedrepo-KD flies display a tendency to decrease the length of their actions (mean bout length) while at the same time increasing the pauses in between actions (interbout interval), with both param- 
A

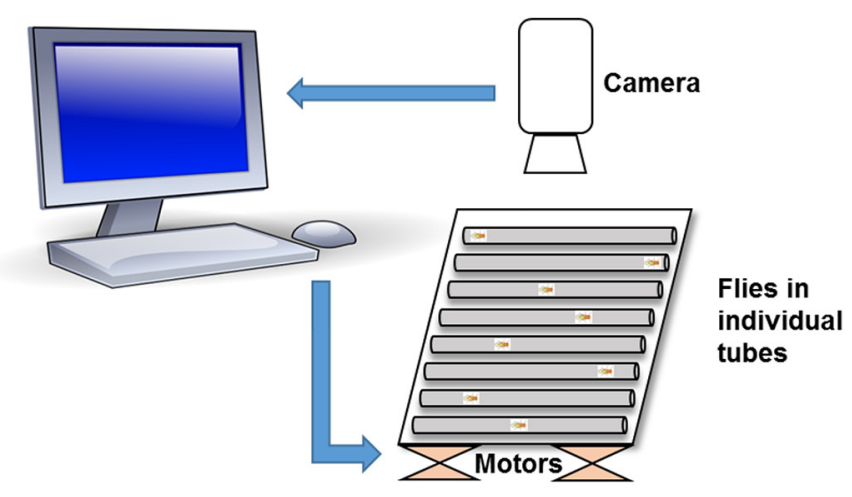

Stimuli Response Protocol

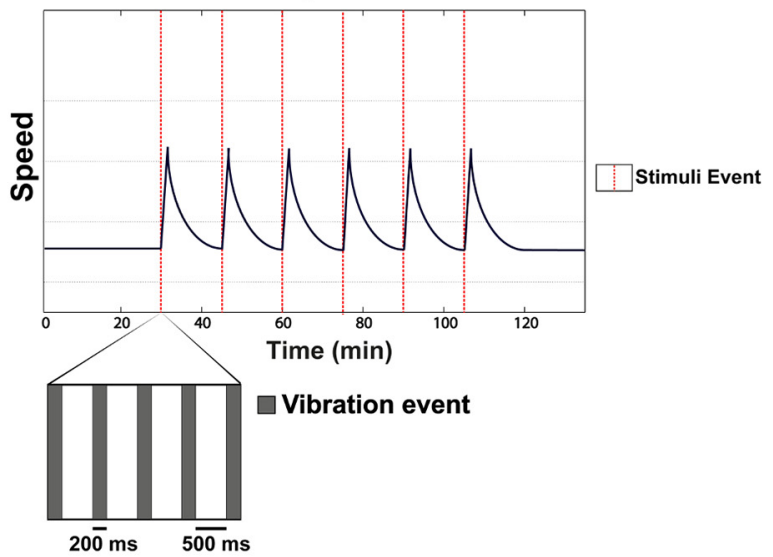

B
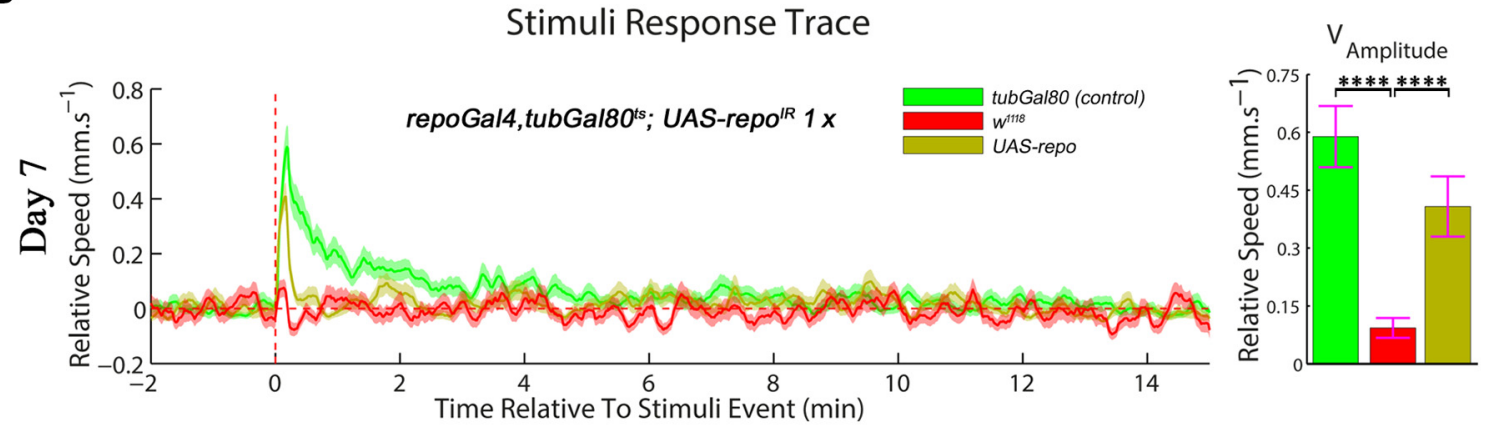

C
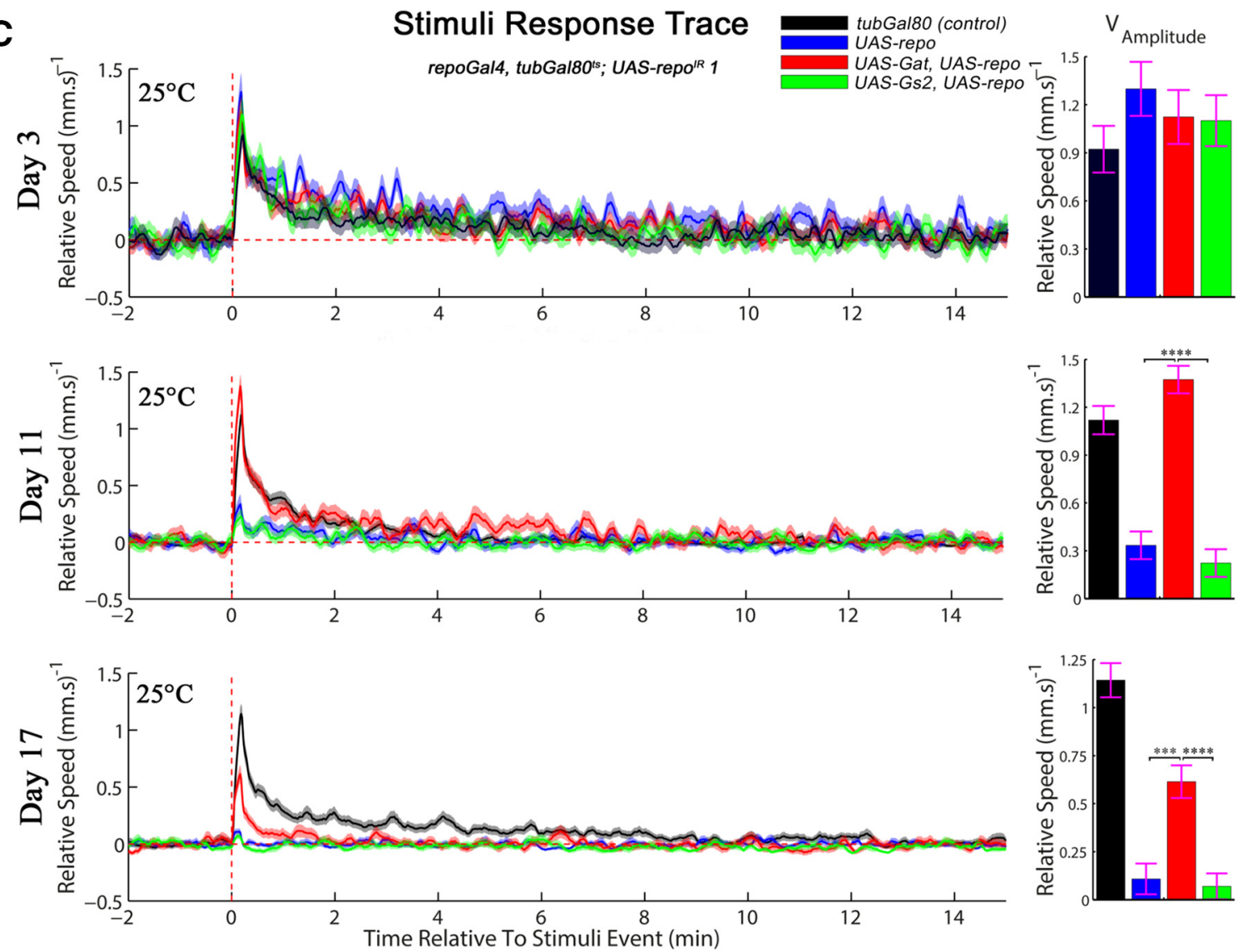

Figure 7. Novel DART paradigm for sophisticated analysis dissects the role of Gat and Gs2 in exogenous motor activity. $\boldsymbol{A}$, Schematic summary of the hardware and behavioral protocol used for the DART stimuli response. See Material and Methods for details of the setup. $\boldsymbol{B}$, Behavioral analysis of the repo-KD flies using an automated setup recording the response to a stimulus at a single fly level. After $7 \mathrm{~d}$ at $29^{\circ} \mathrm{C}$, the repo-KD flies fail to respond to given stimuli (provided by shaft-less motors placed underneath the behavioral platform) and the effect is significantly rescued by the coexpression of a UAS-repo transgene (one-way ANOVA, Dunnett's multiple-comparisons test $p<0.0001$ for both). Twenty flies were assessed per genotype. The (Figure legend continues.) 

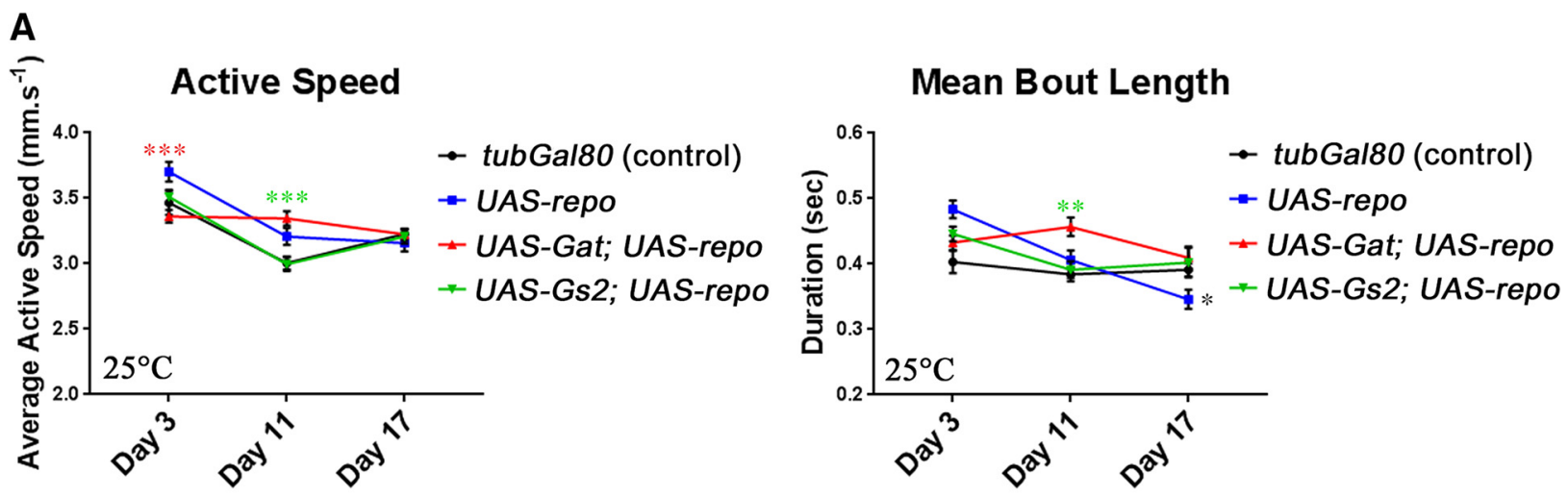

Inter-Bout Interval

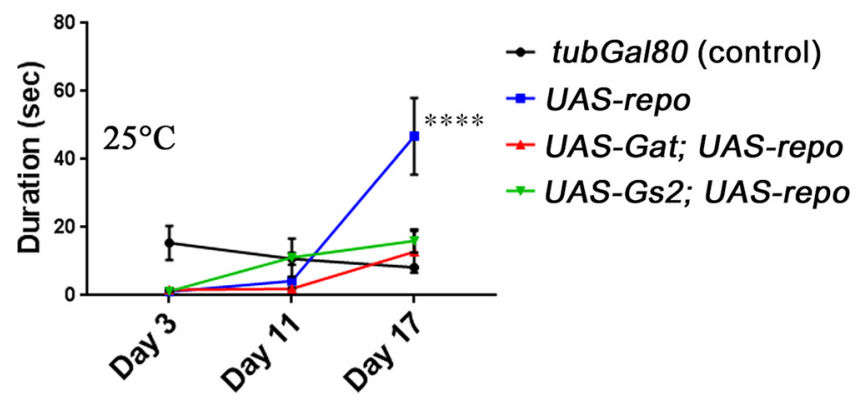

B

Heat-induced seizures
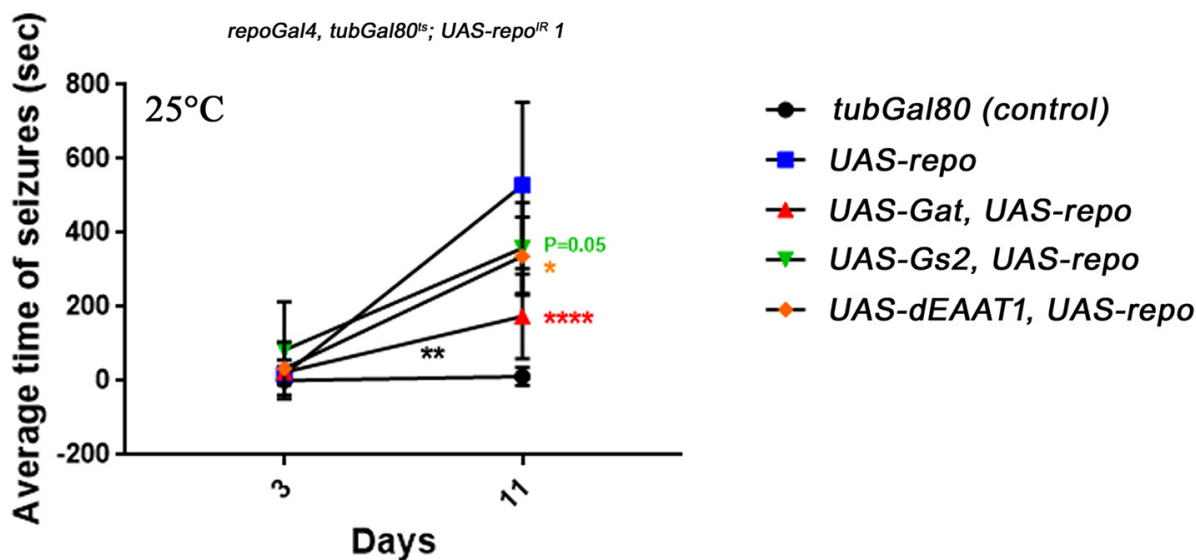

Figure 8. Effect of Gs2 and Gat on endogenous motor activity and recovery from seizures. $A$, Constant tracking of the flies allows the analysis of the endogenously generated motor activity represented here by the three functions active speed, mean bout length, and interbout interval (see Materials and Methods). The flies and data used are the same as in Figure $7 C$. Although the active speed does not seem to be strongly affected, the flies expressing only UAS-repo beside UAS-repo ${ }^{\mathbb{R}} 1$ have a significant reduction in mean bout length after $17 \mathrm{~d}$ and a strong increase of the interbout interval also after $17 \mathrm{~d}$. These changes are not present when either UAS-Gat or UAS-Gs2 is coexpressed. For simplicity, statistical comparisons represented are between UAS-repo vs UAS-Gat + UAS-repo and UAS-repo vs UAS-GS2 + UAS-repo only (two-way ANOVA, Tukey's multiple-comparisonstest $p=0.0004, p=0.0002, p=0.0077, p=0.0300$, and $p<0.0001$, respectively). $\boldsymbol{B}$, Time to recovery analysis of epileptic-like features at 3 and $11 \mathrm{~d}$ at $25^{\circ} \mathrm{C}$. Ten flies were analyzed for each genotype at each time point, repeated five times independently, and averaged. UAS-repo and UAS-Gat co-overexpression significantly rescued the seizure phenotype due to repo knock-down. Black asterisks indicate significance in genotype/time interactions compared with UAS-repo alone (two-way ANOVA, Dunnett's multiple-comparisons test $p=0.0029$ ). Colored asterisks indicate significance between the different genotypes and UAS-repo alone at a specific time point (two-way ANOVA, Dunnett's multiple-comparisons test $p=0.0545, p=0.0248$, and $p<0.0001$, respectively).

\section{$\leftarrow$}

(Figure legend continued.) genotypes were as follows: $w^{1118}$; UAS-repo ${ }^{\mathrm{IR}} 1$; repoGal4, tubGal80 ${ }^{\text {ts }}$ crossed with either: $w^{1118}$; tubGal80 (negative control for the phenotype), $w^{1118}$ or $w^{1118}$; UAS-repo. C, Behavioral analysis of stimuli response (exogenous motor activity) of the same genotypes as in Figure $6 E$, at 3,11 , and $17 \mathrm{~d}$ at $25^{\circ} \mathrm{C}$. Twenty flies were analyzed for each genotype at each time point. The histograms represent the amplitude of the peaks. The coexpression of UAS-Gat and UAS-repo significantly improved the flies' performance compared with UAS-repo alone or UAS-repo and UAS-Gs2 (one-way ANOVA, Dunnett's multiple-comparisons test $p<0.0001$ for both at day 11 and $p=0.0006$ and $p<0.0001$ at day 17.). ${ }^{* * *} p<0.001$, ${ }^{* * * *} p<0.0001$. eters being significantly different from the other genotypes at day 17 (Fig. 8A). Therefore, in addition to a dramatic decrease in response capability, these flies display also a trend toward endogenous inactivity that was highly significant after $17 \mathrm{~d}$ and rescued by UAS-Gat.

Interestingly, coexpression of UAS-Gs2 improved the endogenous mode of activity, with flies coexpressing Gs2 and repo displaying similar mean bout length and interbout intervals to control flies and flies rescued by repo-Gat coexpression (Fig. 8A). However, compared with UAS-Gat, coexpression of UAS-Gs2 failed to provide any 
significant rescue in the response-triggered behavior in the climbing assay and in lifespan (Fig. 6H,I), as well as in the DART setup (Fig. $7 C$ ), indicating a more limited effect for $G s 2$.

In mammals, the orthologes of $d E A A T 1, G s 2$, and Gat are involved in sensitivity to seizures (Pirttimaki et al., 2013; Steinhäuser et al., 2016; Boison and Steinhäuser, 2018) and, recently, a role of $d E A A T 1$ has been reported also in Drosophila (Cho et al., 2018). To test the effect on this important glial function for the same genotypes, we measured the time to recovery using a well established procedure to trigger seizures in flies (Sun et al., 2012). Similar to stimuli-response, we did not observe any significant differences in $3 \mathrm{~d}$ adult flies between all the genotypes assessed (Fig. 8B). However, after $11 \mathrm{~d}$, the rescuedrepo-KD flies developed very long-lasting seizures of up to 25-30 min, with an average time to recovery of $9 \mathrm{~min}$, compared with control flies, which have average seizures of $12 \mathrm{~s}$, similar to the early time point (Fig. $8 B$ ). Interestingly, the coexpression of UAS-Gat improved this defect, whereas UASGs2 and UAS-dEAAT1 did not significantly modify its progression with aging. When considering only the time point at $11 \mathrm{~d}$, UAS-Gat coexpression decreased significantly the time to recovery to $3 \mathrm{~min}$, whereas UAS-Gs2 or UAS-dEAAT1 only mildly affected the phenotype (Fig. $8 B$ ).

Together, these data suggest that the downregulated expression of Gat is, at least partially, specifically responsible for the strong behavioral defects due to the loss of Repo, likely through the alterations of the glutamate/GABA/glutamine cycle and the resulting dramatic reduction in GABA and glutamate levels in the brain.

\section{Discussion}

Repo is specifically needed throughout Drosophila adult life Repo, the key glia determinant in Drosophila, has been mostly studied in embryonic and larval stages, where it is involved in terminal glial differentiation and migration, activating the expression of several genes, some specific to glial cells. Even though constantly expressed in almost all glial cells throughout life and a recognized association of some alleles with neurodegeneration (Xiong and Montell, 1995), its specific role in the adult has largely been neglected, with the exception of a recent study concerning its role in learning and memory (Matsuno et al., 2015).

Here, starting from an miRNA screen, we identified an additional form of regulation of Repo levels through miR-1, having demonstrated in a separate study that this regulation is physiologically relevant in hemocyte development (Trébuchet et al., 2019). Here, we use it ectopically as a discovery tool that validates the ability of miRNA-based screens to inform on the specific relationship with one target gene, even via ectopic miRNA expression.

We show next that repo is continuously required to maintain a viable and fully functional organism because, once its expression is abolished or strongly decreased, repo does not manage to reinstate its initial levels. Indeed, a $3 \mathrm{~d}$ downregulation was enough to irreversibly stop repo expression. Therefore, Repo is continuously required to maintain itself and, thus, a functional nervous system. A model for the molecular basis for this always-ON/always-OFF autoregulation has been proposed in development (Lee and Jones, 2005); however, the simultaneous effects on glial cell fate and number made it difficult to distinguish loss of transcription, from loss of cells. Because such confounding effects are not present in the adult, we provide here unequivocal evidence for repo autoregulation.
Transcriptional regulation of neurotransmitter recycling in adult glial cells affects Drosophila motor activity and recovery from seizures

During glial differentiation, repo triggers the expression of genes such as $d E A A T 1$ and Gs2, both involved in neurotransmitter recycling. Moreover, downregulation of the glial glutamate transporter $d E A A T 1$ and GABA transporter Gat during larval stages triggers dramatic motor defects (Rival et al., 2004; Muthukumar et al., 2014). Here, we show that the transcription of two established direct Repo target genes, dEAAT1 and Gs2 (Kerr et al., 2014), require Repo also during adulthood. Furthermore, we demonstrate for the first time that Repo is also necessary to maintain the expression of the only Drosophila glial GABA transporter, Gat, both at the mRNA and protein levels. However, in the case of Gat, it is likely that its levels are regulated by Repo via an indirect mechanism. No Repo binding sites are present in its promoter and gene sequence, in contrast to $d E A A T 1$ and Gs2. Additional unidentified factors may therefore mediate the effect of Repo on Gat, possibly also through the effect on neurotransmitters levels. Although additional factors may mediate glial subtype specificity, considering that not only astrocytes but also ensheathing glia regulate glutamate homeostasis in Drosophila (Otto et al., 2018), our data strongly suggest that repo is a strictly required topcontroller of the highly conserved glutamate/GABA/glutamine cycle in the Drosophila adult nervous system, governing directly and indirectly the expression of key components.

Consistent with these data, the glial expression of UAS-Gat significantly enhanced the partial rescue by an exogenous UASrepo of the motor defects and epileptic-like features caused by knock-down of the endogenous repo. Althouh neither dEAAT1 nor Gs2 coexpression could rescue all these defects, our genetic setup combining different tools cannot rule out a possible contribution of these two genes to the control of the glutamate/ GABA/glutamine cycle orchestrated by Repo in glia.

To reach these conclusions, we have used a combination of approaches that, from a coarse lifespan analysis, progressed through negative geotaxis responses and to the development of a sophisticated behavioral monitoring system. The key advantage of this approach is that it has allowed us to separate progressively the effect of gene level dysregulation at different levels of refinement, being able to detect events not evident through lifespan analysis or detected much earlier than in simple negative geotaxis assays. This novel behavioral paradigm can therefore be applied successfully to handle more subtle effects in the increasingly popular Drosophila models for neurological disorders.

The precise role of glial GABA transporters in glia is still unclear, considering their potential of clearing GABA from the synapse, but also releasing it extrasynaptically to the postsynaptic neuron as tonic inhibition (Héja et al., 2012). Moreover, even though the glutamate/GABA/glutamine cycle has been extensively studied in mammalian models (Héja et al., 2012; Shameem and Patel, 2012; El-Khoury et al., 2014; Pehrson and Sanchez, 2015; Zheng et al., 2016), the transcriptional regulation of its key genes has been poorly addressed despite its importance in epilepsy, Huntington's disease, and psychiatric disorders (Eid et al., 2013; Huyghe et al., 2014; Cvetanovic et al., 2015; Karki et al., 2015; Boison and Steinhäuser, 2018). This resonates with a wider gap in knowledge of the glial cell functions that contribute to the regulation of neuronal activity via recycling of neurotransmitters. The implication of glia in disease conditions such as epilepsy or psychiatric disorders, where neurotransmitter balance is known to be impaired, has only recently been investigated, particularly for the astrocytic GABA transporters. Indeed, Gat levels in Dro- 
sophila astrocytes are modulated throughout development via metabotropic GABA receptor signaling and Gat regulation can modulate seizure activity (Muthukumar et al., 2014). In addition, TRP channels act on the astrocyte-specific mammalian ortholog GAT-3, regulating its membrane trafficking and/or recycling rate through calcium signaling (Shigetomi et al., 2011). However, nothing is known on transcriptional regulation of GABA transporter genes in mammals. Given their role in epilepsy (Pirttimaki et al., 2013; Schousboe and Madsen, 2017), it is essential to better understand the full extent of their regulation at all levels. The discovery of the importance of repo in maintaining the neurotransmitter balance in the glutamate/GABA/glutamine cycle point toward the importance of understanding the transcriptional regulation, and could provide a useful and tractable model to unravel the glial contribution in human disease with neurotransmitter imbalance.

\section{References}

Auld VJ, Fetter RD, Broadie K, Goodman CS (1995) Gliotactin, a novel transmembrane protein on peripheral glia, is required to form the bloodnerve barrier in Drosophila. Cell 81:757-767.

Baron O, Boudi A, Dias C, Schilling M, Nölle A, Vizcay-Barrena G, Rattray I, Jungbluth H, Scheper W, Fleck RA, Bates GP, Fanto M (2017) Stall in canonical autophagy-lysosome pathways prompts nucleophagy-based nuclear breakdown in neurodegeneration. Curr Biol 27:3626-3642.e6.

Bartel DP (2009) MicroRNAs: target recognition and regulatory functions. Cell 136:215-233.

Bejarano F, Bortolamiol-Becet D, Dai Q, Sun K, Saj A, Chou YT, Raleigh DR, Kim K, Ni JQ, Duan H, Yang JS, Fulga TA, Van Vactor D, Perrimon N, Lai EC (2012) A genome-wide transgenic resource for conditional expression of Drosophila microRNAs. Development 139:2821-2831.

Boison D, Steinhäuser C (2018) Epilepsy and astrocyte energy metabolism. Glia 66:1235-1243.

Chaturvedi R, Reddig K, Li HS (2014) Long-distance mechanism of neurotransmitter recycling mediated by glial network facilitates visual function in Drosophila. Proc Natl Acad Sci U S A 111:2812-2817.

Cho S, Muthukumar AK, Stork T, Coutinho-Budd JC, Freeman MR (2018) Focal adhesion molecules regulate astrocyte morphology and glutamate transporters to suppress seizure-like behavior. Proc Natl Acad Sci U S A 115:11316-11321.

Cvetanovic M, Ingram M, Orr H, Opal P (2015) Early activation of microglia and astrocytes in mouse models of spinocerebellar ataxia type 1. Neuroscience 289:289-299.

Daniels RW, Gelfand MV, Collins CA, DiAntonio A (2008) Visualizing glutamatergic cell bodies and synapses in Drosophila larval and adult CNS. J Comp Neurol 508:131-152.

Eid T, Tu N, Lee TS, Lai JC (2013) Regulation of astrocyte glutamine synthetase in epilepsy. Neurochem Int 63:670-681.

El-Khoury R, Panayotis N, Matagne V, Ghata A, Villard L, Roux JC (2014) GABA and glutamate pathways are spatially and developmentally affected in the brain of Mecp2-deficient mice. PLoS One 9:e92169.

Faville R, Kottler B, Goodhill GJ, Shaw PJ, van Swinderen B (2015) How deeply does your mutant sleep? Probing arousal to better understand sleep defects in Drosophila. Sci Rep 5:8454.

Flici H, Cattenoz PB, Komonyi O, Laneve P, Erkosar B, Karatas OF, Reichert H, Berzsenyi S, Giangrande A (2014) Interlocked loops trigger lineage specification and stable fates in the Drosophila nervous system. Nat Commun 5:4484.

Furrer SA, Mohanachandran MS, Waldherr SM, Chang C, Damian VA, Sopher BL, Garden GA, La Spada AR (2011) Spinocerebellar ataxia type 7 cerebellar disease requires the coordinated action of mutant ataxin-7 in neurons and glia, and displays non-cell-autonomous Bergmann glia degeneration. J Neurosci 31:16269-16278.

Halter DA, Urban J, Rickert C, Ner SS, Ito K, Travers AA, Technau GM (1995) The homeobox gene repo is required for the differentiation and maintenance of glia function in the embryonic nervous system of Drosophila melanogaster. Development 121:317-332.

Héja L, Nyitrai G, Kékesi O, Dobolyi A, Szabó P, Fiáth R, Ulbert I, Pál-Szenthe B, Palkovits M, Kardos J (2012) Astrocytes convert network excitation to tonic inhibition of neurons. BMC Biol 10:26.
Huyghe D, Nakamura Y, Terunuma M, Faideau M, Haydon P, Pangalos MN, Moss SJ (2014) Glutamine synthetase stability and subcellular distribution in astrocytes are regulated by gamma-aminobutyric type $B$ receptors. J Biol Chem 289:28808-28815.

Karki P, Kim C, Smith K, Son DS, Aschner M, Lee E (2015) Transcriptional regulation of the astrocytic excitatory amino acid transporter 1 (EAAT1) via NF-kappaB and yin yang 1 (YY1). J Biol Chem 290:23725-23737.

Kerr KS, Fuentes-Medel Y, Brewer C, Barria R, Ashley J, Abruzzi KC, Sheehan A, Tasdemir-Yilmaz OE, Freeman MR, Budnik V (2014) Glial Wingless/ Wnt regulates glutamate receptor clustering and synaptic physiology at the Drosophila neuromuscular junction. J Neurosci 34:2910-2920.

Klaes A, Menne T, Stollewerk A, Scholz H, Klämbt C (1994) The ets transcription factors encoded by the Drosophila gene pointed direct glial cell differentiation in the embryonic CNS. Cell 78:149-160.

Kottler B, Fiore VG, Ludlow ZN, Buhl E, Vinatier G, Faville R, Diaper DC, Stepto A, Dearlove J, Adachi Y, Brown S, Chen C, Solomon DA, White KE, Humphrey DM, Buchanan SM, Sigrist SJ, Endo K, Ito K, de Bivort B, et al. (2017) A lineage-related reciprocal inhibition circuitry for sensorymotor action selection. bioRxiv. Advance online publication. Retrieved January 15, 2017. doi:10.1101/100420.

Kozomara A, Griffiths-Jones S (2011) miRBase: integrating microRNA annotation and deep-sequencing data. Nucleic Acids Res 39:D152-D157.

Lee BP, Jones BW (2005) Transcriptional regulation of the Drosophila glial gene repo. Mech Dev 122:849-862.

Lim LP, Lau NC, Garrett-Engele P, Grimson A, Schelter JM, Castle J, Bartel DP, Linsley PS, Johnson JM (2005) Microarray analysis shows that some microRNAs downregulate large numbers of target mRNAs. Nature 433: 769-773.

Lynch AM, Murphy KJ, Deighan BF, O’Reilly JA, Gun'ko YK, Cowley TR, Gonzalez-Reyes RE, Lynch MA (2010) The impact of glial activation in the aging brain. Aging Dis 1:262-278.

Marvin JS, Borghuis BG, Tian L, Cichon J, Harnett MT, Akerboom J, Gordus A, Renninger SL, Chen TW, Bargmann CI, Orger MB, Schreiter ER, Demb JB, Gan WB, Hires SA, Looger LL (2013) An optimized fluorescent probe for visualizing glutamate neurotransmission. Nat Methods 10:162-170

Matsuno M, Horiuchi J, Yuasa Y, Ofusa K, Miyashita T, Masuda T, Saitoe M (2015) Long-term memory formation in Drosophila requires trainingdependent glial transcription. J Neurosci 35:5557-5565.

Muthukumar AK, Stork T, Freeman MR (2014) Activity-dependent regulation of astrocyte GAT levels during synaptogenesis. Nat Neurosci 17: $1340-1350$.

Napoletano F, Occhi S, Calamita P, Volpi V, Blanc E, Charroux B, Royet J, Fanto M (2011) Polyglutamine atrophin provokes neurodegeneration in Drosophila by repressing fat. EMBO J 30:945-958.

Nisoli I, Chauvin JP, Napoletano F, Calamita P, Zanin V, Fanto M, Charroux B (2010) Neurodegeneration by polyglutamine atrophin is not rescued by induction of autophagy. Cell Death Differ 17:1577-1587.

Otto N, Marelja Z, Schoofs A, Kranenburg H, Bittern J, Yildirim K, Berh D, Bethke M, Thomas S, Rode S, Risse B, Jiang X, Pankratz M, Leimkühler S, Klämbt C (2018) The sulfite oxidase shopper controls neuronal activity by regulating glutamate homeostasis in Drosophila ensheathing glia. Nat Commun 9:3514.

Pehrson AL, Sanchez C (2015) Altered gamma-aminobutyric acid neurotransmission in major depressive disorder: a critical review of the supporting evidence and the influence of serotonergic antidepressants. Drug Des Devel Ther 9:603-624.

Pirttimaki T, Parri HR, Crunelli V (2013) Astrocytic GABA transporter GAT-1 dysfunction in experimental absence seizures. J Physiol 591: 823-833.

Rae C, Hare N, Bubb WA, McEwan SR, Bröer A, McQuillan JA, Balcar VJ, Conigrave AD, Bröer S (2003) Inhibition of glutamine transport depletes glutamate and GABA neurotransmitter pools: further evidence for metabolic compartmentation. J Neurochem 85:503-514.

Rival T, Soustelle L, Strambi C, Besson MT, Iché M, Birman S (2004) Decreasing glutamate buffering capacity triggers oxidative stress and neuropil degeneration in the Drosophila brain. Curr Biol 14:599-605.

Roth FC, Draguhn A (2012) GABA metabolism and transport: effects on synaptic efficacy. Neural Plast 2012:805830.

Rowley NM, Madsen KK, Schousboe A, Steve White H (2012) Glutamate and GABA synthesis, release, transport and metabolism as targets for seizure control. Neurochem Int 61:546-558. 
Schousboe A, Madsen KK (2017) Delineation of the role of astroglial GABA transporters in seizure control. Neurochem Res 42:2019-2023.

Schwabe T, Bainton RJ, Fetter RD, Heberlein U, Gaul U (2005) GPCR signaling is required for blood-brain barrier formation in Drosophila. Cell 123:133-144.

Shameem M, Patel AB (2012) Glutamatergic and GABAergic metabolism in mouse brain under chronic nicotine exposure: implications for addiction. PLoS One 7:e41824.

Shigetomi E, Tong X, Kwan KY, Corey DP, Khakh BS (2011) TRPA1 channels regulate astrocyte resting calcium and inhibitory synapse efficacy through GAT-3. Nat Neurosci 15:70-80.

Silver SJ, Hagen JW, Okamura K, Perrimon N, Lai EC (2007) Functional screening identifies miR-315 as a potent activator of wingless signaling. Proc Natl Acad Sci U S A 104:18151-18156.

Sinakevitch I, Grau Y, Strausfeld NJ, Birman S (2010) Dynamics of glutamatergic signaling in the mushroom body of young adult Drosophila. Neural Dev 5:10.

Sokol NS, Ambros V (2005) Mesodermally expressed Drosophila microRNA-1 is regulated by twist and is required in muscles during larval growth. Genes Dev 19:2343-2354.

Steinhäuser C, Grunnet M, Carmignoto G (2016) Crucial role of astrocytes in temporal lobe epilepsy. Neuroscience 323:157-169.

Stork T, Sheehan A, Tasdemir-Yilmaz OE, Freeman MR (2014) Neuron- glia interactions through the heartless FGF receptor signaling pathway mediate morphogenesis of Drosophila astrocytes. Neuron 83:388-403.

Sun L, Gilligan J, Staber C, Schutte RJ, Nguyen V, O’Dowd DK, Reenan R (2012) A knock-in model of human epilepsy in Drosophila reveals a novel cellular mechanism associated with heat-induced seizure. J Neurosci 32:14145-14155.

Trébuchet G, Cattenoz PB, Zsámboki J, Mazaud D, Siekhaus DE, Fanto M, Giangrande A (2019) The repo homeodomain transcription factor suppresses hematopoiesis in Drosophila and preserves the glial fate. J Neurosci 39:238-255.

Xiong WC, Montell C (1995) Defective glia induce neuronal apoptosis in the repo visual system of Drosophila. Neuron 14:581-590.

Xiong WC, Okano H, Patel NH, Blendy JA, Montell C (1994) repo encodes a glial-specific homeo domain protein required in the Drosophila nervous system. Genes Dev 8:981-994.

Yuasa Y, Okabe M, Yoshikawa S, Tabuchi K, Xiong WC, Hiromi Y, Okano H (2003) Drosophila homeodomain protein REPO controls glial differentiation by cooperating with ETS and BTB transcription factors. Development 130:2419-2428.

Zheng Y, Yang Y, Dong B, Zheng H, Lin X, Du Y, Li X, Zhao L, Gao H (2016) Metabonomic profiles delineate potential role of glutamate-glutamine cycle in $\mathrm{db} / \mathrm{db}$ mice with diabetes-associated cognitive decline. Mol Brain 9:40. 